\title{
Suppression of Internal Wave Breaking in the Antarctic Circumpolar Current near Topography
}

\author{
STEPHANIE WATERMAN* \\ National Oceanography Centre, University of Southampton, Southampton, United Kingdom \\ KURT L. POLZIN \\ Woods Hole Oceanographic Institution, Woods Hole, Massachusetts \\ Alberto C. Naveira Garabato, Katy L. Sheen, and Alexander Forryan \\ National Oceanography Centre, University of Southampton, Southampton, United Kingdom
}

(Manuscript received 17 August 2012, in final form 8 November 2013)

\begin{abstract}
Simultaneous full-depth microstructure measurements of turbulence and finestructure measurements of velocity and density are analyzed to investigate the relationship between turbulence and the internal wave field in the Antarctic Circumpolar Current. These data reveal a systematic near-bottom overprediction of the turbulent kinetic energy dissipation rate by finescale parameterization methods in select locations. Sites of near-bottom overprediction are typically characterized by large near-bottom flow speeds and elevated topographic roughness. Further, lower-than-average shear-to-strain ratios indicative of a less near-inertial wave field, rotary spectra suggesting a predominance of upward internal wave energy propagation, and enhanced narrowband variance at vertical wavelengths on the order of $100 \mathrm{~m}$ are found at these locations. Finally, finescale overprediction is typically associated with elevated Froude numbers based on the near-bottom shear of the background flow, and a background flow with a systematic backing tendency. Agreement of microstructure- and finestructure-based estimates within the expected uncertainty of the parameterization away from these special sites, the reproducibility of the overprediction signal across various parameterization implementations, and an absence of indications of atypical instrument noise at sites of parameterization overprediction, all suggest that physics not encapsulated by the parameterization play a role in the fate of bottom-generated waves at these locations. Several plausible underpinning mechanisms based on the limited available evidence are discussed that offer guidance for future studies.
\end{abstract}

\section{Introduction}

Recent studies report on the microstructure observations of the turbulent kinetic energy dissipation rate $\epsilon$ and finestructure observations of internal wave-scale flow properties across different regimes of the Antarctic Circumpolar Current (ACC) collected as part of the

\footnotetext{
* Current affiliation: Department of Earth, Ocean, and Atmospheric Sciences, University of British Columbia, Vancouver, Canada.

Corresponding author address: Stephanie Waterman, Department of Earth, Ocean, and Atmospheric Sciences, University of British Columbia, 2207 Main Mall, Vancouver, BC V6T 1Z4 Canada.

E-mail: snw@alum.mit.edu
}

Southern Ocean Finestructure (SOFine) and the Diapycnal and Isopycnal Mixing Experiment in the Southern Ocean (DIMES) campaigns (Waterman et al. 2013; Sheen et al. 2013). These observations allow the first direct studies of the relationship between turbulence and the internal wave field in the deep ACC and have provided evidence of enhanced near-bottom turbulent dissipation in association with strong near-bottom flows, rough topography, and regions where the internal wave field is found to have enhanced energy, less inertial frequency content, and a dominance of upward-propagating energy. As such, the data provide strong support for the view that deep turbulent dissipation and mixing in the Southern Ocean are primarily underpinned by the breaking of internal waves generated as deep-reaching geostrophic flows impinge on rough seafloor topography, 
a view that has been suggested by a number of previous indirect studies (e.g., Naveira Garabato et al. 2004; Sloyan 2005; Kunze et al. 2006; Nikurashin and Ferrari 2010a; Wu et al. 2011).

These studies also reveal common, thought-provoking discrepancies between various theoretical expectations and observed quantities. First, both analyses find that the observed deep enhancement of turbulent dissipation over small-scale topography is not as pronounced as expected based on the anticipated lee-wave energy flux for the observed near-bottom flow speeds, stratification, and seafloor roughness. Second, both find that the observed near-bottom turbulent dissipation rates are, in key locations, systematically low relative to estimates of downscale energy transfer via finescale parameterization formulas characterizing nonlinear wave-wave interactions. Both discrepancies between anticipated and observed values are found to be up to an order of magnitude in regions prone to bottom wave generation, that is, regions of relatively large topographic roughness and near-bottom flow speeds.

There exist a number of possible explanations for the observed discrepancies. One possible explanation is the assumption of two-dimensional isotropic topography in the lee-wave theory and as such its neglect of nonlinear flow-splitting effects. These effects may be significant in Drake Passage where the topographic steepness parameter $\epsilon=h N / U$, where $h$ is the characteristic topographic height and $U / N$ is the characteristic vertical scale of lee waves given by the ratio of the mean velocity $U$ and the buoyancy frequency $N$ at the ocean bottom, is estimated to be 0.7-0.8 (Nikurashin and Ferrari 2010a). However, we do not expect the same for the SOFine region, where the steepness parameter is typically small [estimated to be 0.1-0.2 in Waterman et al. (2013)]. Alternatively, the mismatch may point to a nonlocal dissipation of the radiated energy owing to the waves' horizontal and vertical propagation and the horizontal advection of the generating flow. Another possible explanation is that the mismatch results from a nondissipative fate for a fraction of the energy in the radiated wave field. The observed spatial variability in the mismatch between the finestructure-derived estimates of the dissipation rate and the corresponding microstructure estimates provides some evidence in support of these latter two scenarios. In key locations, a significant portion of the unaccounted for energy is present in the local internal wave field, but it appears not to have "cascaded down" to the turbulent scales (Sheen et al. 2013). While the mechanism(s) that underpin the discrepancy remain unclear, such results pose intriguing questions regarding the generation and evolution of waves forced by the ACC impinging on rough topography.
The purpose of this paper is to examine the relation between microstructure estimates of the turbulent dissipation rate and those predicted by various implementations of the finescale parameterization in the SOFine data in detail. We evaluate the potential roles of parameterization biases, instrumentation issues, and physics key to wave generation and evolution in underpinning the systematic near-bottom finescale overprediction signal that we observe. After making a case for a physical interpretation of the overprediction signal in this dataset, we speculate on possible underpinning mechanisms and evaluate their relevance to the extent the available data permit. Although the available observations do not permit a definitive test of the various hypotheses, they do present useful evidence to guide future exploration of the phenomenon.

The outline of this paper is as follows: In section 2, we briefly describe the data that form the basis of our study and outline the analysis performed to derive the microstructure and finestructure estimates of $\epsilon$. Here we also briefly present the physical basis of the finescale parameterization used to predict the dissipation rate associated with internal wave breaking from finestructure measurements, as well as the various practical implementations of the parameterization that we consider in this work. Our discussion of the finescale parameterization here is not intended to serve as a complete review. See Polzin et al. (2014) for this purpose. In section 3, we present the results of our study documenting in detail 1) the tendency of the finescale parameterization to overpredict the microstructure-derived estimate of the turbulent dissipation rate near the bottom in key places and the robustness of this signal to parameterization implementation issues and instrumental noise; and 2) the dependence of the microstructure-finestructure relationship on various physical factors key to bottom wave generation and evolution and characteristics of the observed internal wave field. In section 4 , we discuss possible explanations for the observed near-bottom finescale overprediction and present observational clues that can help to evaluate their relevance and guide future study.

\section{Data and analysis}

\section{a. Data}

Our study uses observations from the SOFine project, an observational process study examining turbulence, its underpinning processes, and its larger-scale dynamics in a mixing hotspot in the Southern Ocean. Involving the first full-depth microstructure measurements in the Southern Ocean, the SOFine observations make a direct study of turbulence and its relationship to the internal wave field in the deep ACC possible for the first time. 

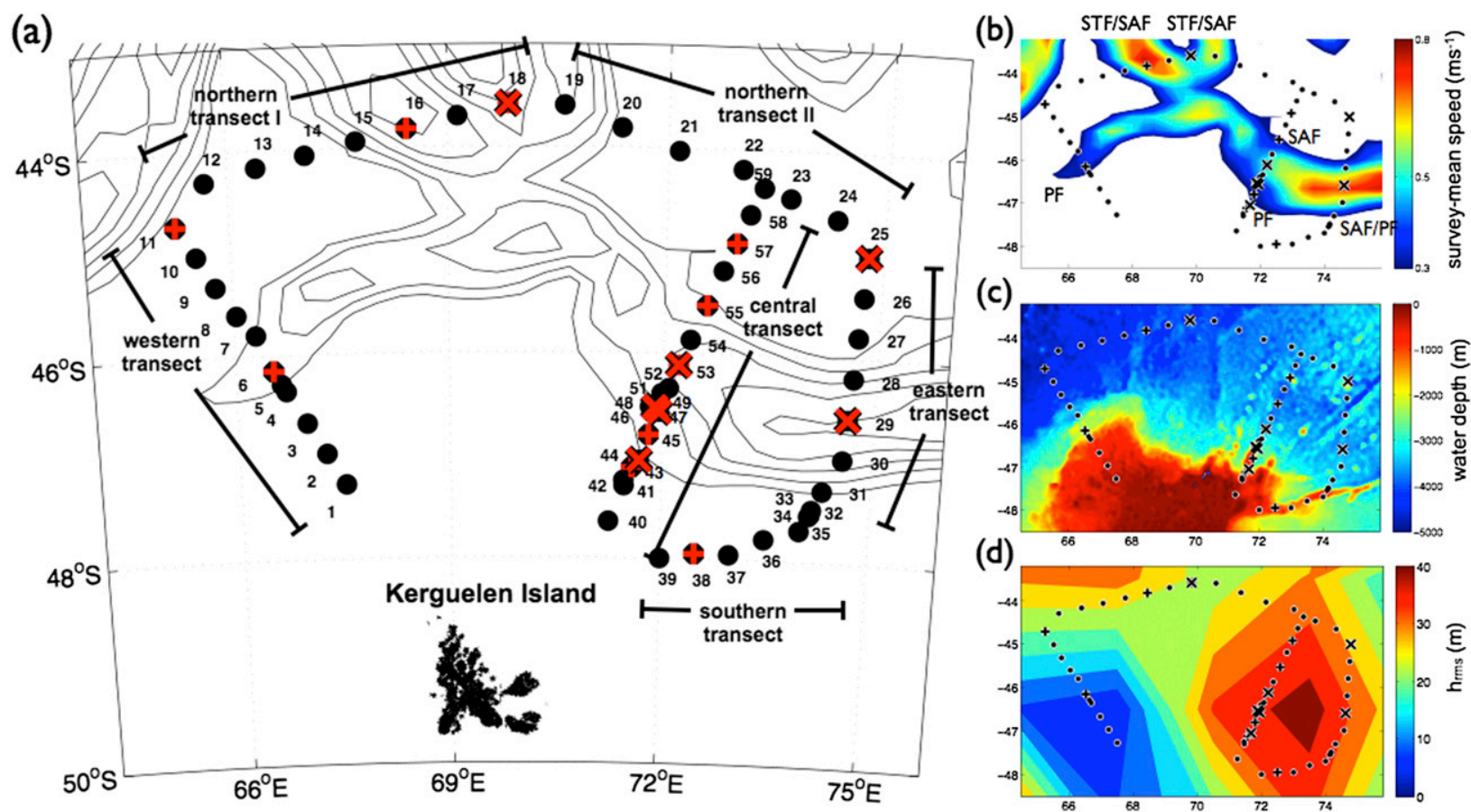

FIG. 1. (a) Map of the SOFine survey. Black contours show the survey-mean surface geostrophic flow speed from Archiving, Validation, and Interpretation of Satellite Oceanographic data (AVISO) altimetry and are drawn at intervals of $10 \mathrm{~cm} \mathrm{~s}^{-1}$ in the range of $30-80 \mathrm{~cm} \mathrm{~s}^{-1}$. (b) The survey-mean surface geostrophic flow speed in the region from AVISO altimetry with the intersecting branches of the Polar Front (PF), Subantarctic Front (SAF), and Subtropical Front (STF) indicated. (c) Regional large-scale bathymetry at 1-min resolution from Smith and Sandwell ship-sounding bathymetry, version 14.1 (Smith and Sandwell 1997). (d) An estimate of regional small-scale topographic roughness from Nikurashin and Ferrari (2011). In all panels, stations with an average finescale overprediction in the bottommost $1000 \mathrm{~m}$ are indicated by plus signs. The eight special stations with above average near-bottom finescale overprediction are indicated by larger crosses.

A detailed description of the SOFine site and observations is given in Waterman et al. (2013). In brief, the SOFine field campaign was situated on the northern flank of the Kerguelen Plateau in the south Indian Ocean, a region where two main jets of the ACC form large standing meanders in climatological atlases and ocean circulation models alike (Sparrow et al. 1996; Sokolov and Rintoul 2009). It is also a region of complex topography expected to host elevated levels of internal wave activity and turbulence as strong near-bottom flows impinge on the roughness of the plateau slopes (e.g., Nikurashin and Ferrari 2011; Scott et al. 2011; Naveira Garabato et al. 2013). The SOFine survey, observations from which are considered here, was conducted in November and December 2008 and consisted of a double box survey defined by three sections emanating from the Kerguelen Plateau closed by a land boundary (Kerguelen Island) to the south and a perpendicular transect to the north of the plateau slopes (Fig. 1). At the time of the survey, these sections intersected various ACC frontal jets (Fig. 1b). Survey station measurements included microstructure measurements of the centimeter-scale shear made by a vertical microstructure profiler (VMP), from which the dissipation rate of turbulent kinetic energy was estimated as $\epsilon=(15 / 2) \nu(\partial u / \partial z)^{2}$ (here $\nu$ is the molecular viscosity, isotropy is assumed, and $\partial u / \partial z$ represents one of two vertical gradients of horizontal velocity). Simultaneously, finestructure measurements of internal wave-scale shear and strain were made from conductivity-temperaturedepth (CTD) and lowered acoustic Doppler current profiler (LADCP) instruments. Sampling at all stations and for all instruments spanned the full water depth to within a nominal $\sim 10 \mathrm{~m}$ of the ocean floor. For further details, see Naveira Garabato (2009) and Waterman et al. (2013).

\section{b. Analysis}

\section{1) Microstructure}

Microstructure processing was done by the authors using algorithms developed for the High Resolution Profiler (Polzin and Montgomery 1996) modified to acknowledge the different sensor and noise characteristics of the VMP. We also used software provided by Rockland Scientific International, the manufacturer of the VMP. In 
the former case, estimates of the gradient variances were made at 0.5 - $\mathrm{dB}$ vertical resolution by Fourier-transforming piece lengths of the shear record of $1 \mathrm{~s}$ with a sampling rate of $512 \mathrm{~Hz}$, then defining a spectral minimum representing the intersection of signal (turbulence) and noise (electronic and vibrational). In the latter case, multiple piece lengths were used to estimate the portion of the shear record coherent with adjacent threedimensional accelerometer data. This coherent signal was interpreted as vibrational contamination of the shear record and subtracted. The two methods return estimates of gradient variance that are consistent for signal levels in excess of the vibrational contamination.

\section{2) Finestructure}

CTD data were processed at 2-dB vertical resolution using Sea-Bird Electronics (SBE) software (SBE Data Processing, version 7.18). LADCP data were processed both at $10-\mathrm{m}$ vertical resolution using the velocityinversion method [Lamont-Doherty Earth Observatory (LDEO) software, version IX6; see Visbeck (2002)] and at 5-m vertical resolution using the "shear method" [University of Hawaii Data Acquisition System Common Ocean Data Access System (UHDAS + CODAS) software, version 2011.12.30; see Firing and Gordon (1990), Fischer and Visbeck (1993), and King et al. (2001)]. The former method incorporates bottom-track information and as such provides robust estimates of absolute velocity near the bottom. Data processed using the latter method were used for estimates of vertical shear owing to the method's direct shear calculation.

Finestructure results presented here focus on predictions of the turbulent dissipation rate from the application of the finescale parameterization to CTD and LADCP measurements of strain and shear, and in particular to vertical spectra of these quantities integrated between specified wavelengths. Strain, a measure of the stretching and squeezing of isopycnal layers, was calculated using the local relative change in buoyancy frequency from a background value $\zeta_{z}=\left(N^{2}-N_{\text {ref }}^{2}\right) / N_{\text {ref }}^{2}$. The buoyancy frequency $N$ was calculated from the CTD estimates of temperature and salinity using the Commonwealth Scientific and Industrial Research Organisation (CSIRO) seawater routines (SeaWater library, version 3.0). The background value $N_{\text {ref }}$ was computed by adiabatic leveling over a pressure range of $400 \mathrm{~dB}$ to calculate a reference $N$ profile at each station. Shear, the vertical gradient of the horizontal flow velocity $V_{z}$, was calculated as the first difference of the vertical profiles of horizontal velocity measured by the LADCP. Vertical wavenumber spectra of buoyancy frequency-normalized shear and strain were calculated by segmenting the CTD and LADCP profile data into common half-overlapping vertical segments of $640 \mathrm{~m}$. Segments were constructed both starting from the surface and the bottom, with near-surface values computed from the vertical segments defined starting from the surface, near-bottom values computed from the vertical segments defined starting from the bottom, and interior values computed from an average of top-down- and bottom-up-defined segments. In this way, each spectral calculation contains a full number of points; that is, we avoid zero padding at the surface and bottom boundary bins is avoided. CTD data were interpolated onto a $2.5-\mathrm{m}$ depth grid prior to Fourier-transforming segments of 256 points, so that the first 128 wavenumbers coincided with those in the Fourier-transformed LADCP data segments of 128 points. The segmented data were detrended (by removing a linear fit) and windowed with a Hanning window of the segment length before Fourier transformation, and resulting spectra were compensated for the loss of variance from windowing. For the typical estimates, strain spectra were corrected for bin-to-bin first differencing, while the shear spectra were corrected for loss of variance due to range averaging, finite differencing, interpolation, and instrument tilting. See Polzin et al. (2002) for further details. We ignore the correction for the horizontal smoothing resulting from beam spreading, which Polzin et al. (2002) found to be a minor effect. Results using uncorrected spectra were also considered to ensure the robustness of our conclusions. In the case of the LADCP profile data, spectra were computed for the down- and upcast profiles separately, and then averaged before integration.

\section{3) Finescale Parameterization IMPLEMENTATIONS}

The finescale parameterization of the turbulent dissipation rate $\epsilon_{\text {fine }}$ characterizes the downscale energy transfer associated with nonlinear wave-wave interactions at vertical scales on the order of $10-100 \mathrm{~m}$. Currently used finestructure algorithms (e.g., Gregg et al. 2003; Kunze et al. 2006) are derivatives of the finestructure parameterization originally presented in Polzin et al. (1995) (Polzin et al. 2014). That formula summarizes the energy transport associated with raytracing simulations as

$$
F(m, \omega)=A\left(\frac{\omega^{2}-f^{2}}{N^{2}-\omega^{2}}\right)^{1 / 2} N^{-1} m E(m, \omega) \int_{0}^{m_{c}} m^{\prime 2} E_{k}\left(m^{\prime}\right) d m^{\prime},
$$

where $F(m, \omega)$ represents the spectral energy transport in the vertical wavenumber domain integrated over the horizontal azimuth domain. Here $A=0.20$, an empirical 
nondimensional constant that sets the transport magnitude taken from validation studies (e.g., Gregg 1989; Polzin et al. 1995); $\omega$ is the wave intrinsic frequency; $f$ is the inertial frequency; $m$ is the vertical wavenumber; $E(m, \omega)$ is the energy density; and $E_{k}(m)$ is its kinetic energy component. The high-wavenumber limit $m_{c}$ represents the scale associated with the transition into wave-breaking phenomena. The link to the dissipation rate is made through a radiation balance equation, which relates this spectral energy transport to the rates of turbulent production, dissipation, and diapycnal mixing. See Polzin et al. (2014) for further details.

Equation (1) expresses the physical basis of the finescale parameterization. However, in practice, the finescale observations to which the parameterization is typically applied provide only incomplete information on the two-dimensional wavenumber-frequency spectrum $E(m, \omega)$, and various approximations in both the vertical wavenumber and frequency domains are hence required. Integration over the internal wave frequency domain in the absence of knowledge of the frequency content of the wave field (as is often the case for coarse temporal sampling) introduces a correction dependent on the only available source of information on the frequency content of the wave field, the ratio of horizontal kinetic and potential energies. This ratio is often known as the shear-to-strain ratio $R_{\omega}$ and also links the total and kinetic energy densities. Evoking a local closure for the shear variance in the vertical wavenumber domain, that is, $\int_{0}^{m} m^{2} E_{k}\left(m^{\prime}\right) d m^{\prime} \cong m^{3} E_{k}(m)$, allows further simplification, although it assumes a flat [Garrett-Munk (GM) internal wave spectrum like] spectral shape (Munk 1981; Gregg and Kunze 1991). The resulting socalled local approximation to Eq. (1), invoking an $R_{\omega}$ dependence to represent the effects of the wave field frequency content, then simplifies to

$F(m)=A \frac{f}{f_{0}} \frac{\cosh ^{-1}\left(\frac{N}{f}\right)}{\cosh ^{-1}\left(\frac{N_{0}}{f_{0}}\right)} \sqrt{\frac{2}{R_{\omega}-1}} N^{-1} E(m) m^{4} E_{k}(m)$.

The finescale parameterization used in common practice is derived from Eqs. (1) and (2). For reasons of convenience, it is formulated by reference to the GM internal wave spectrum rather than directly using the above equations. Further, when the instrumentation does not resolve $m_{c}$ and/or the parameterization is applied to individual profiles for which the transport estimates via Eqs. (1) and (2) can be noisy, the integration bounds in Eq. (1) of $\left(0, m_{c}\right)$ are replaced with bounds of $\left(m_{1}, m_{2}\right)$ to estimate the average shear and strain spectral density in this vertical wavenumber band. Here again, for this simplification to be valid, a flat spectral shape is assumed. By identifying $2 m^{2} E_{k}(m)$ as the vertical shear spectrum $V_{z}^{2}$ and implementing these various practical changes to Eq. (1), one arrives at the more common implementation [as applied, e.g., in Kunze et al. (2006)]

$$
\epsilon_{\text {fine }}=\epsilon_{0} \frac{\frac{\left\langle\overline{V_{z}^{2}}\right\rangle}{\bar{N}^{2}}}{\frac{\left\langle V_{z \mathrm{GM}}^{2}\right\rangle}{N_{0}^{2}}} h\left(R_{\omega}\right) L(\theta, N)
$$

Here $\epsilon_{0}=7.8 \times 10^{-10} \mathrm{~W} \mathrm{~kg}^{-1}$ and is the background turbulent dissipation of the GM internal wave spectrum at latitude $30^{\circ}$ and in stratification $N_{0}=5.24 \times$ $10^{-3} \mathrm{rad} \mathrm{s}^{-1}$. Angle brackets represent an average in the vertical wavenumber range $\left(m_{1}, m_{2}\right)$, so that $\left\langle\overline{V_{z}^{2}}\right\rangle=$ $1 /\left(m_{2}-m_{1}\right) \int_{m_{1}}^{m_{2}} V_{z}^{2}(m) d m$. The functions $h\left(R_{\omega}\right)$ and $L(\theta, N)$ account for the wave frequency and latitudinal dependence and are described in Polzin et al. (1995) and Gregg et al. (2003), respectively.

The choice of the integration limits $\left(m_{1}, m_{2}\right)$ is a potential source of bias in the finescale parameterization estimate if either the wave field does not fit the model (GM) shape as is assumed in the derivation of Eqs. (2) and (3), or if instrument performance prevents an accurate characterization of the spectral shape of the wave field over the wavenumber band considered. As such, in our implementation of the finescale parameterization described by Eq. (3), we consider different sets of integration limits used by various authors in past finestructure studies. Specifically, these include 1) a fixed range of integration limits common to both LADCP and CTD data; 2) a fixed range of integration limits with different ranges for LADCP and CTD data; and $3)$ variable ranges of integration with different ranges for LADCP and CTD data and lower limits of integration determined by noise criteria. The use of different integration ranges for LADCP and CTD data aims to avoid small scales in the LADCP variance estimate where the LADCP data become noisy, and large scales in the CTD variance estimate where strain variance is less likely to originate from internal waves. However, this implementation requires normalizing by the variance in the GM model spectrum integrated over the same wavenumber band and as such an assumption that the gradient spectra have the same shape as the GM model. The GM model spectrum and parameters used here are those of the 1976 version of the Garrett and Munk spectrum [GM76 model; see Polzin and Lvov (2011) for discussion of that model and its variants]. In the case of integration limits set 1 (our "standard" estimate), 
we choose very conservative (i.e., large) vertical wavelengths as the common limits of integration, specifically $\lambda_{z \min }=130 \mathrm{~m}$ and $\lambda_{z \max }=320 \mathrm{~m}$. These scales are significantly larger than the short wavelengths where LADCP noise appears to impact the mean spectral variance levels (see appendix A for a discussion). In the case of integration limits set 2 the choice of the shear and strain integration bandwidth is taken from Kunze et al. (2006); GM-normalized LADCP shear variances are integrated between vertical wavelengths of 130 and $320 \mathrm{~m}$ (to avoid instrument noise at smaller wavelengths) and GM-normalized CTD strain variances are integrated between vertical wavelengths of 30 and $150 \mathrm{~m}$ (to avoid contamination by background stratification). In the instance of set 3 , we apply different integration ranges for the LADCP and CTD data as in set 2 , but in each case with a variable lower limit of integration decided on a spectrum-by-spectrum basis determined by a criterion based on noise considerations. In the case of the CTD data, as in Kunze et al. (2006) and following the concerns of Gargett (1990) about underestimating the internal wave variance if the spectrum becomes saturated at vertical wavelengths $\lambda_{z}$ greater than $10 \mathrm{~m}$, we use a lower limit given by the shortest wavelength for which $\int_{\lambda_{z \min }}^{150 \mathrm{~m}} \Im\left(\zeta_{z}^{2}\right)\left(\lambda_{z}\right) d \lambda_{z}<0.1$ (where $\Im$ denotes a GM-normalized spectrum) or $10 \mathrm{~m}$, using whichever is larger. In the case of the LADCP data, we consider the noise model of Polzin et al. (2002) and set the minimum wavelength of integration to the larger of $130 \mathrm{~m}$ and the minimum wavelength for which the noise spectral level is less than a critical ratio (taken to be 0.33 in the results presented here) times the observed shear spectral level. We also consider implementations of Eqs. (1) and (2) to examine the finescale parameterization prediction independent of the choice of these integration limits.

\section{Results}

\section{a. Near-bottom finescale parameterization overprediction and its relation to implementation issues and instrumental noise}

We compare the microstructure-derived estimates of the turbulent dissipation rate with those predicted by various implementations of the finescale parameterization, an exercise that reveals a robust tendency for nearbottom finescale overprediction in special locations. This observation of a robust finescale overprediction signal is the motivation for our study. The signal is illustrated by visualizing the logarithm of the ratio of the dissipation rate predicted by the parameterization $\epsilon_{\text {fine }}$ to that derived from microstructure measurements $\epsilon_{\text {micro }}$ (hereafter the $\epsilon$ ratio). This overprediction is shown for a typical implementation of the finescale parameterization [our standard estimate given by Eq. (3) with a fixed range of integration limits common to both LADCP and CTD data] for the SOFine survey observations in Fig. 2. This visualization reveals a systematic near-bottom overprediction by the parameterization (hereafter finescale overprediction) in key places, typically inside the ACC jets (Fig. 1) and where the near-bottom dissipation rate is high (Fig. 2a).

Near-bottom overprediction is seen in the stationaveraged vertical profile of the $\epsilon$ ratio as a function of height above bottom (shown for this particular implementation of the parameterization in Fig. 3). This average profile shows finescale overprediction by a factor of $2.3 \pm 0.4$ on average in the bottommost $1500 \mathrm{~m}$. However, as Figs. 1 and 2 indicate, the tendency for near-bottom overprediction is not a widespread phenomenon, but instead is localized to specific sites. As such, it is more meaningful to consider this signal in a select subset of stations. We consider the group of eight stations that show anomalously high near-bottom overprediction, specifically above average finescale overprediction relative to all stations with average finescale overprediction in the bottommost $1000 \mathrm{~m}$. The average $\epsilon$ ratio profile for this subset of stations (Fig. 3, black line) indicates that for these "special" sites the average finescale overprediction in the bottommost $1500 \mathrm{~m}$ is $5.1 \pm 0.5$, with individual stations that form this average often showing an off-bottom overprediction at a particular height that is an order of magnitude or more (see Fig. 14, described in greater detail below, and the discussion). These values should be contrasted to the overall average $\epsilon$ ratio value between 1500- and 3000-m height and the equivalent near-bottom average $\epsilon$ ratio value for the nonspecial sites, which are $1.0 \pm 0.2$ and $1.5 \pm 0.5$, respectively, both well within the expected factor of 2 uncertainty of finescale parameterization estimates (Gregg 1989; Polzin et al. 1995). The success of the parameterization in predicting the dissipation rate away from the near-bottom and away from these special sites gives confidence in both the general quality of the microstructure and finestructure data and in our implementation of the parameterization. It points to something systematic at these special locations.

Further confidence in the robustness of the overprediction signal is provided by the fact that this nearbottom finescale overprediction is a reproducible feature in various implementations of the parameterization. These include implementations that consider smaller wavelengths of integration for strain and that use variable ranges of integration for both shear and strain with lower limits of integration determined by noise criteria 
(a)
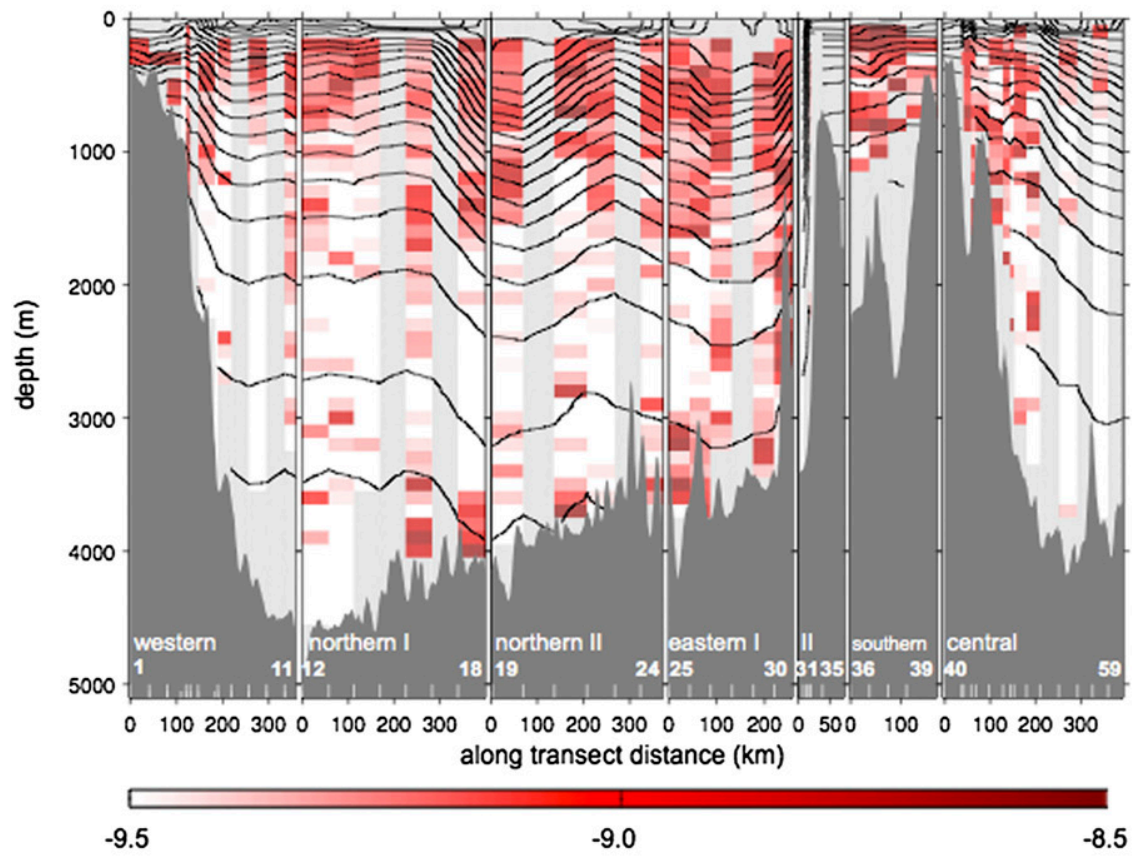

$\log _{10}\left(\varepsilon_{\text {micro }}\right)\left(\mathrm{Wkg}^{-1}\right)$

(b)

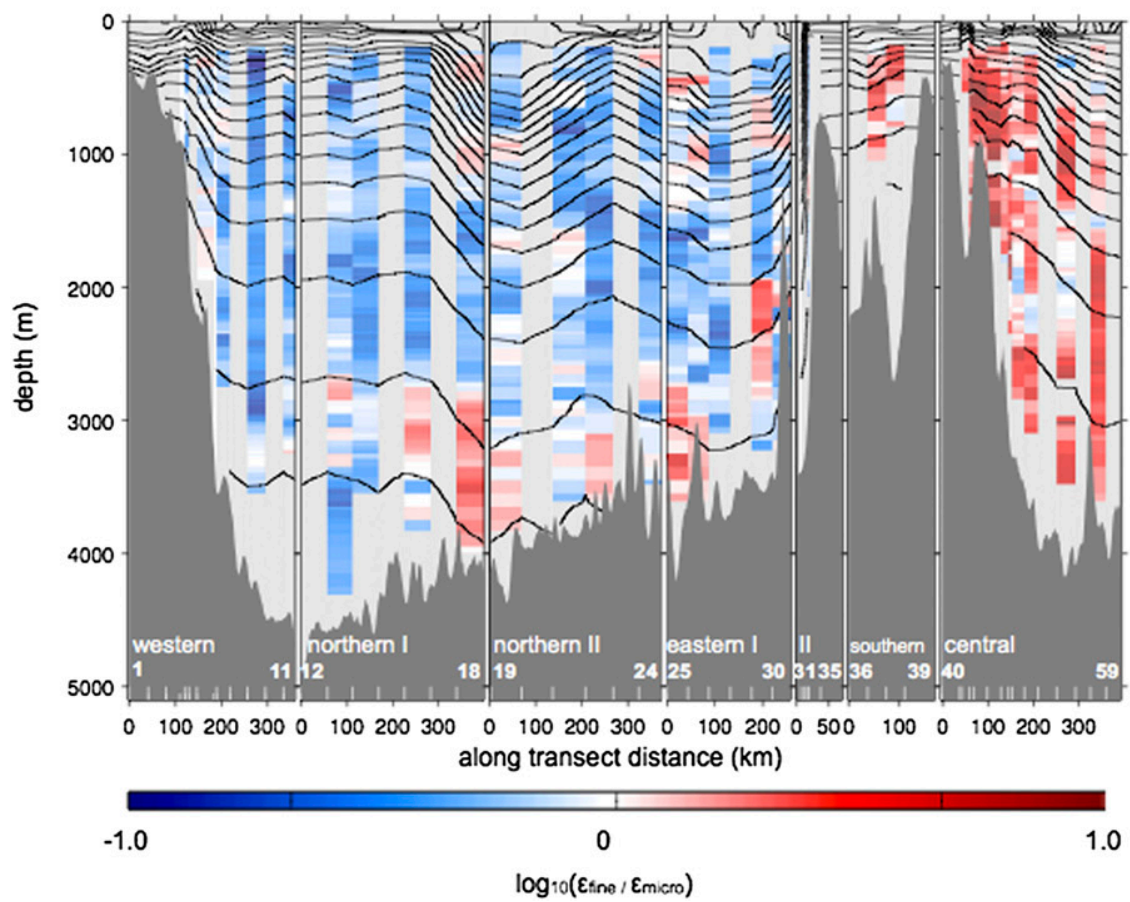

FIG. 2. An along-transect distance-depth section of (a) $\epsilon_{\text {micro }}$ and (b) the $\epsilon$ ratio $=\epsilon_{\text {fine }} / \epsilon_{\text {micro }}$, both displayed on a logarithmic scale. The section, as displayed, starts in the southwestern corner of the survey domain, then runs clockwise along the rim of the region, and finally runs northeastward along the central transect, with each subsection corresponding to an individual transect as indicated. White ticks at the bottom of (a) and (b) delineate individual stations with key station numbers labeled to help orient the reader. Neutral density contours in the range of $26-28.4 \mathrm{~kg} \mathrm{~m}^{-3}$ in $0.1 \mathrm{~kg} \mathrm{~m}^{-3}$ intervals are shown by the black contours. Bottom topography is from Smith and Sandwell ship-sounding bathymetry version 14.1 (Smith and Sandwell 1997). 


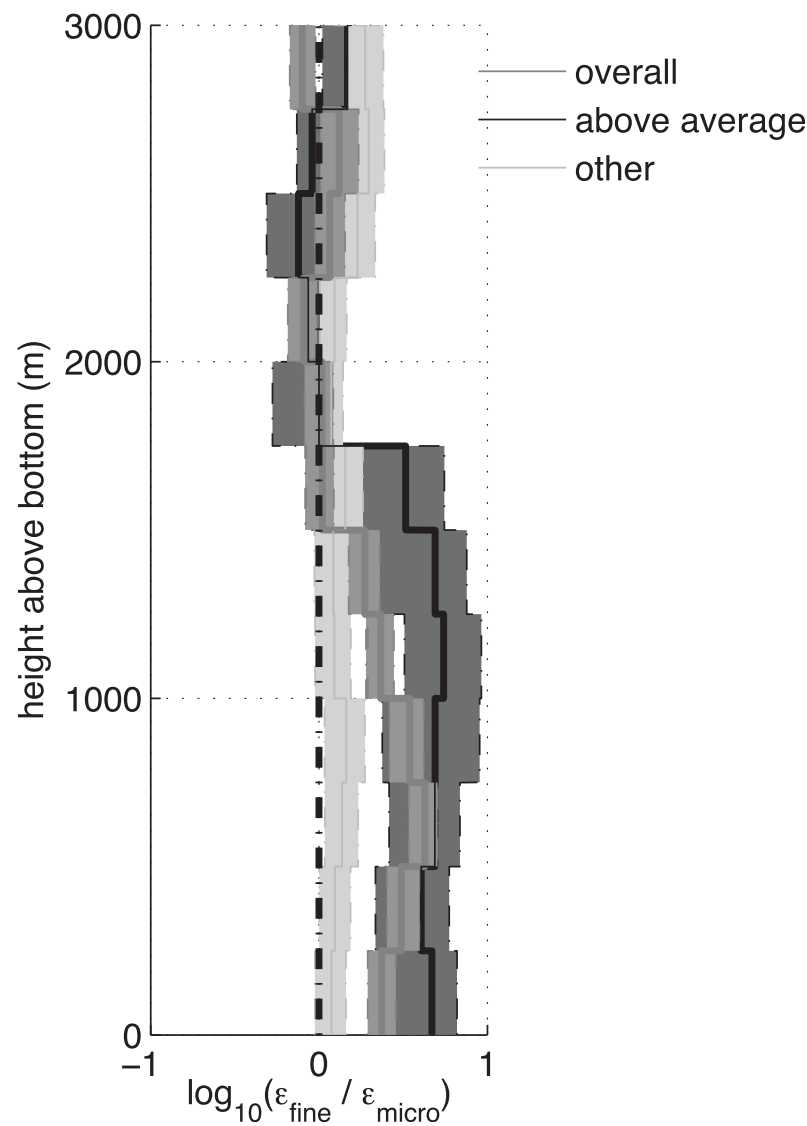

FIG. 3. The station-averaged vertical profile of the $\epsilon$ ratio as a function of height above the bottom. The overall (all station) mean (dark gray) is contrasted with the average from a subset group of eight stations that show above average near-bottom finescale overprediction (see text for details; black) and the average from the remaining stations (light gray). The shaded areas show the $90 \%$ confidence interval calculated by bootstrapping.

as described in section 2; implementations that do not employ the spectral corrections of Polzin et al. (2002); and implementations that use strain-only information (as, e.g., in Kunze et al. 2006). The gross spatial patterns of finescale overprediction revealed in our standard implementation displayed in Fig. 2 are repeated in all of these various implementations (Fig. 4), and the tendency for overprediction in the bottommost $1500 \mathrm{~m}$ in a station-averaged sense is a robust feature of all finescale parameterization implementations considered (Fig. 5).

To describe the $\epsilon_{\text {fine }}-\epsilon_{\text {micro }}$ relationship for the various implementations quantitatively and to evaluate whether the overprediction seen in near-bottom data at the special sites is significant, we perform linear regressions of the various $\epsilon_{\text {fine }}$ estimates on the local average value of $\epsilon_{\text {micro }}$ in the corresponding $\epsilon_{\text {fine }}$ transform interval. Results are displayed in Table 1 . The calculation assumes a linear model $\epsilon_{\text {fine }}=m \epsilon_{\text {micro }}+b$, and as such the slope $m$ indicates a bulk measure of the degree of finescale under or overprediction, while the intercept $b$ indicates a representative constant offset of the $\epsilon_{\text {fine }}$ prediction relative to the local average $\epsilon_{\text {micro }}$ value. We evaluate the goodness of fit of such a model by computing the $R$ squared statistic and its associated $P$ value suitable for testing the hypothesis of no correlation. An effective number of degrees of freedom for the correlation calculation is computed based on the integral length scale of the $\epsilon_{\text {fine }}$ profile in relation to the total profile length on a station-by-station basis. The $P$ values displayed correspond to a correlation calculation, assuming the mean effective number of degrees of freedom over all stations. We consider the linear regression coefficients $m$ and $b$ as well as the statistical metrics of $R$ squared and $P$ for a calculation that uses the full dataset (all stations and all depths below $500 \mathrm{~m}$ ) and one that uses a subset of data corresponding to the special eight stations at and below 1500-m height above bottom only (numbers in parentheses).

The results displayed in Table 1 indicate first that all finescale parameterization estimates provide a good description of the range of microstructure-derived dissipation rates observed when the dataset is considered as a whole. Shear- and strain-based parameterization estimates are characterized by $m$ values in the range of 1.0 1.8 within $95 \%$ confidence interval bounds and as such are within the anticipated factor of 2 uncertainty of the parameterization estimate. Intercepts $b$ are not distinguishable from zero within these same confidence bounds. The $R$ square values are in the range of 0.6-0.7, and $P$ values are in the range of $0.01-0.06$. Strain-only based estimates perform slightly less well with respect to predicting the observed microstructure values (with $m$ values in the range of 2-4, lower $R$ squared values, and higher $P$ values), potentially a symptom of an increased significance of the bias arising from the inclusion of quasi-permanent finestructure at larger wavelengths and/or the failure to account for the frequency content of the wave field through an $R_{\omega}$ dependence. Still, the general agreement for all the implementations considered gives further confidence in the quality of the measurements and in our implementations of the parameterization.

Contrasting these statistics with those computed for the subset of data from near the bottom at the special sites (Table 1, numbers in parentheses) shows a statistically significant difference in the $\epsilon_{\text {fine }}-\epsilon_{\text {micro }}$ relationship. Regression slope estimates for these calculations are typically 3 or 4 and everywhere greater than the expected factor of 2 to a $95 \%$ confidence level. This metric of overprediction is up to an order of magnitude for the 

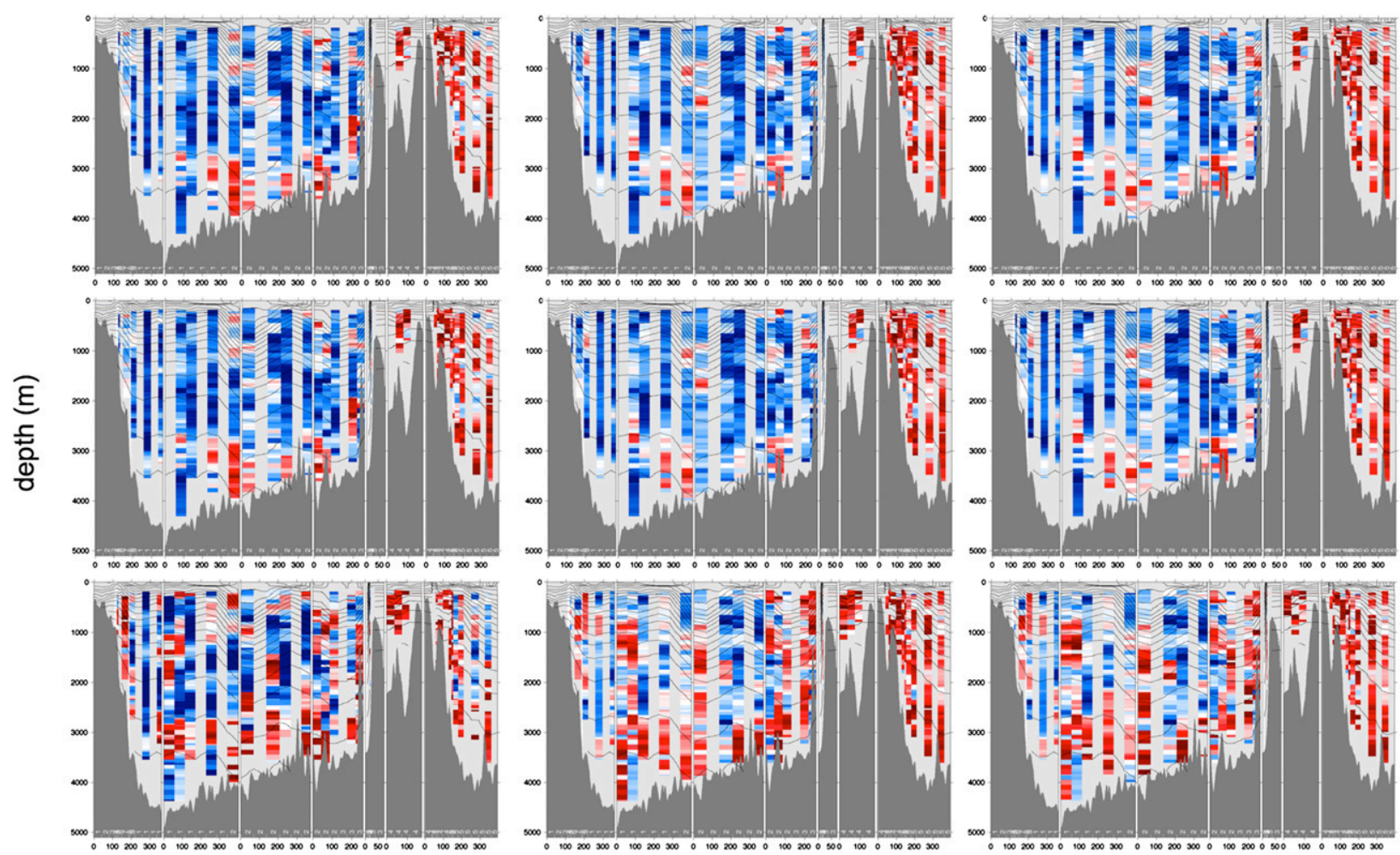

along transect distance $(\mathrm{km})$

$$
-1.0
$$

0

$$
\log _{10}\left(\frac{\varepsilon_{\text {fine }}}{\varepsilon_{\text {micro }}}\right)
$$

FIG. 4. Visualizations of the $\epsilon$ ratio for various implementations of the finescale parameterization (top) with spectral corrections as described in the text applied to both shear and strain spectra; (middle) with no spectral corrections applied to shear and strain spectra; (bottom) using corrected strain data only and assuming a constant $R_{\omega}$ value of 7; (left) employing a fixed range of integration limits common to both shear and strain data, $\lambda_{z \min }=130 \mathrm{~m}$ and $\lambda_{z \max }=320 \mathrm{~m}$ (hereafter limits 1 ); (center) using a fixed range of integration limits with different ranges for LADCP and CTD data, 130-320 m for GM-normalized shear variance and 30-150 m for GM-normalized strain variance (hereafter limits 2); and (right) using variable ranges of integration with different ranges for LADCP and CTD data and lower limits of integration determined by noise criteria as described in the text (hereafter limits 3 ).

strain-only parameterization estimates. At the same time, we note that estimates of correlation and our confidence in its significance are in fact higher for these select cases, likely a result of the fact that we tend to observe finescale overprediction at locations where the dissipation rate is high and presumably where signalto-noise ratios are more favorable. The finding that all implementations of the finescale parameterization considered do a good job of describing the microstructurederived dissipation rate generally, yet also consistently show a robust and statistically significant tendency for overprediction at the special locations, provides some support for a physical (as opposed to instrumental or implementation based) interpretation of the overprediction signal. The presence of a near-bottom finescale overprediction signal in the strain-only estimates in particular suggests that contamination by LADCP instrument noise, an issue that tends to lead to an overestimation of the shearinferred dissipation rates in regions of weak stratification (Kunze et al. 2006), does not explain the bulk of the signal. This is consistent with Sheen et al. (2013), who also document a near-bottom overprediction signal in strainonly estimates. It is nonetheless prudent to consider the dependence of the finescale overprediction observed on metrics of instrumental noise and the vertical wavelength integration limits in detail.

\section{1) RELATiOn to instrumental NOISE}

As the signature of finescale overprediction occurs at depth in association with weak stratification and 


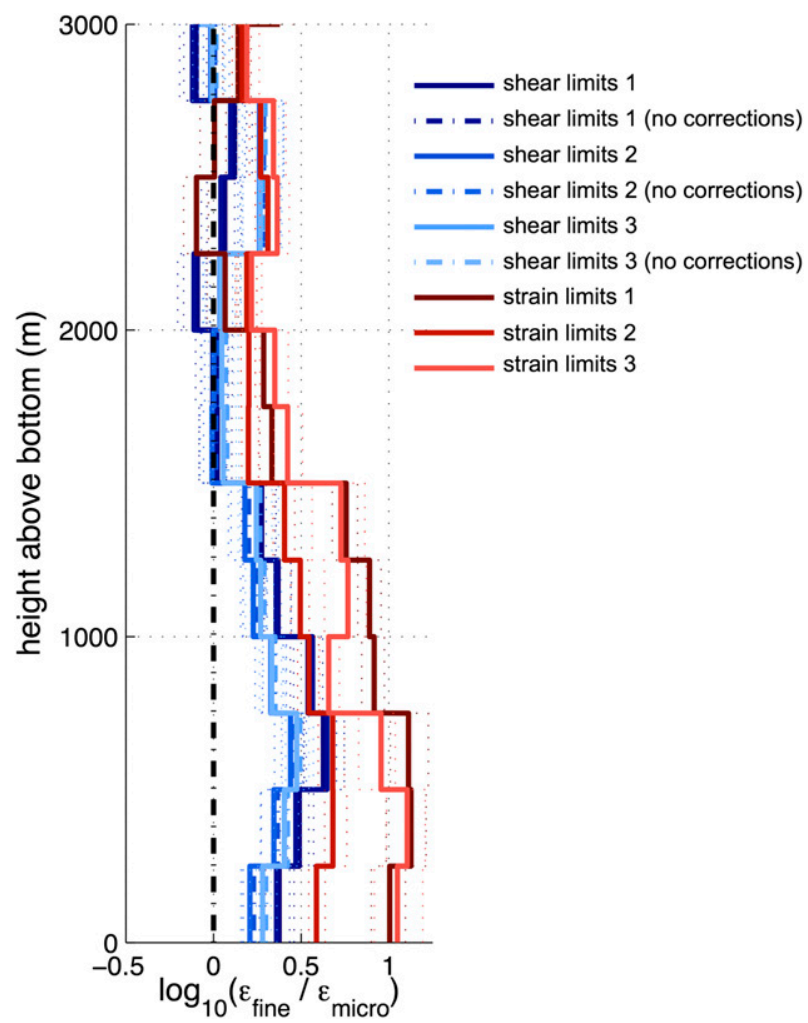

FIG. 5. A comparison of the station-averaged vertical profiles of the $\epsilon$ ratio as a function of height above bottom for various implementations of the finescale parameterization described in the caption of Fig. 4. The dotted lines show the $90 \%$ confidence interval calculated by bootstrapping.

typically lower LADCP signal-to-noise ratios, noise contaminating the shear variance estimate is of particular concern (Kunze et al. 2006; Polzin et al. 2014). We have devoted appendix A to describing the LADCP noise characteristics in this dataset, and here we document an absence of any indication of atypical instrument noise in association with regions of greatest finescale overprediction. Instead, locations of enhanced $\epsilon_{\text {micro }}-\epsilon_{\text {fine }}$ discrepancy tend to be associated with lower LADCP noise levels than those that characterize the dataset as a whole. Further, the observed stratification is neither extremely low nor are the profile segments exhibiting finescale overprediction associated with anomalously high shear-to-strain ratios (see Fig. 14, described in greater detail below). If anything, the opposite is true. Both findings suggest that LADCP noise is not the key factor underpinning the finescale overprediction documented here.

An alternative source of the $\epsilon_{\text {micro }}-\epsilon_{\text {fine }}$ discrepancy is of course a low bias to the microstructure estimates at the sites of finescale overprediction. To evaluate this possibility, we consider diagnostics of the microstructure data quality near the bottom at the eight special sites that exhibit the most pronounced $\epsilon_{\text {micro }}-\epsilon_{\text {fine }}$ deviations. Figure 6 presents a standard diagnostic summary plot of bin-averaged microstructure shear spectra from these locations with best-fit Nasmyth spectra superimposed. We find that estimates of shear variance from the two independent probes are consistent, that the shear spectra are well resolved, and that the observed spectra agree with the Nasmyth spectra characterizing high Reynolds number turbulence. Further, we find that the estimates of dissipation from both microstructure processing methods we employ are consistent. These facts give confidence in the robustness of the microstructure observations at these locations.

\section{2) RELATION TO VERTICAL SCALES CONSIDERED}

The repeated near-bottom overprediction signal across various finescale parameterization implementations suggests robustness of the overprediction signal to the choice for the vertical wavelength limits of integration. It does not, however, address the potential for the overprediction to derive from the application of the parameterization to scales larger or smaller than those to which the physics of the parameterization is expected to apply. We note that the finescale parameterization is constructed around a cascade of energy from large to small vertical scales, with $m=m_{c}$ being the highwavenumber terminus of the cascade. For waves with $m \simeq m_{c}$, nonlinear transports are sufficiently vigorous as to remove energy near $m=m_{c}$ in several wave periods. At larger vertical scales, however, linear wave propagation and wave-mean flow interactions become increasingly important relative to nonlinearity. At smaller vertical scales, energy can be transported directly to turbulent production scales by other mechanisms such as shear instabilities.

We investigate the possibility of bias arising from the choice of integration limits by examining the forms of both the nominal ray-tracing formula [Eq. (1)] as well as its local approximation [Eq. (2)] as a function of vertical wavenumber for the group of stations with aboveaverage near-bottom overprediction (Fig. 7). The visualization reveals that finescale overprediction is robust at $m=m_{c}$ for the wavenumber-independent standard implementation, the wavenumber-dependent nominal ray-tracing formula described by Eq. (1), and its local approximation described by Eq. (2), with the ratio of the average finescale prediction to the average microstructure estimate of the dissipation rate being 10,12, and 7 , respectively. We note that as shear and strain at locations showing finescale overprediction tend to have relatively elevated spectral levels, they also have smaller 
TABLE 1. Results of a linear regression of $\epsilon_{\text {fine }}$ on $\epsilon_{\text {micro }}$ for various implementations of the finescale parameterization as described in the text and the caption of Fig. 4. Statistics displayed correspond to results using the full dataset (all stations, all depths below $500 \mathrm{~m}$ ); the numbers in parentheses indicate the corresponding statistic for the calculation using data from the special eight stations at and below 1500-m height above bottom only. The range in slope and intercept indicates a $95 \%$ confidence interval.

\begin{tabular}{|c|c|c|c|c|}
\hline & $m$ & $b\left(\times 10^{-9} \mathrm{~W} \mathrm{~kg}^{-1}\right)$ & $R$ squared & $P$ \\
\hline Shear and strain (corrected, limits 1) & $\begin{array}{c}1.6 \pm 0.2 \\
(3.3 \pm 0.6)\end{array}$ & $\begin{array}{c}-0.1 \pm 0.2 \\
(-0.5 \pm 0.6)\end{array}$ & $\begin{array}{c}0.6 \\
(0.9)\end{array}$ & $\begin{array}{c}0.06 \\
(<0.01)\end{array}$ \\
\hline Shear and strain (corrected, limits 2) & $\begin{array}{c}1.3 \pm 0.1 \\
(3.4 \pm 0.7)\end{array}$ & $\begin{array}{c}-0.1 \pm 0.1 \\
(-0.9 \pm 0.7)\end{array}$ & $\begin{array}{c}0.7 \\
(0.7)\end{array}$ & $\begin{array}{c}0.01 \\
(<0.01)\end{array}$ \\
\hline Shear and strain (corrected, limits 3) & $\begin{array}{c}1.2 \pm 0.2 \\
(4.6 \pm 0.9)\end{array}$ & $\begin{array}{c}0.0 \pm 0.2 \\
(1.3 \pm 0.9)\end{array}$ & $\begin{array}{c}0.6 \\
(0.9)\end{array}$ & $\begin{array}{c}0.05 \\
(<0.01)\end{array}$ \\
\hline Shear and strain (uncorrected, limits 1) & $\begin{array}{c}1.6 \pm 0.2 \\
(3.2 \pm 0.6)\end{array}$ & $\begin{array}{c}-0.1 \pm 0.2 \\
(-0.5 \pm 0.6)\end{array}$ & $\begin{array}{c}0.7 \\
(0.8)\end{array}$ & $\begin{array}{c}0.01 \\
(<0.01)\end{array}$ \\
\hline Shear and strain (uncorrected, limits 2) & $\begin{array}{c}1.3 \pm 0.1 \\
(3.2 \pm 0.6)\end{array}$ & $\begin{array}{c}-0.1 \pm 0.1 \\
(-0.8 \pm 0.7)\end{array}$ & $\begin{array}{c}0.6 \\
(0.9)\end{array}$ & $\begin{array}{c}0.06 \\
(<0.01)\end{array}$ \\
\hline Shear and strain (uncorrected, limits 3) & $\begin{array}{c}1.2 \pm 0.2 \\
(4.5 \pm 0.8)\end{array}$ & $\begin{array}{c}0.2 \pm 0.2 \\
(-1.3 \pm 0.8)\end{array}$ & $\begin{array}{c}0.6 \\
(0.9)\end{array}$ & $\begin{array}{c}0.05 \\
(<0.01)\end{array}$ \\
\hline Strain only (corrected, limits 1 ) & $\begin{array}{c}2.5 \pm 0.9 \\
(9 \pm 2)\end{array}$ & $\begin{array}{c}2 \pm 2 \\
(-0.8 \pm 3)\end{array}$ & $\begin{array}{c}0.3 \\
(0.8)\end{array}$ & $\begin{array}{c}0.04 \\
(<0.01)\end{array}$ \\
\hline Strain only (corrected, limits 2) & $\begin{array}{c}2.4 \pm 0.1 \\
(4.4 \pm 0.6)\end{array}$ & $\begin{array}{c}-0.1 \pm 0.2 \\
(-1.1 \pm 0.8)\end{array}$ & $\begin{array}{c}0.4 \\
(0.9)\end{array}$ & $\begin{array}{c}0.1 \\
(<0.01)\end{array}$ \\
\hline Strain only (corrected, limits 3) & $\begin{array}{l}4.1 \pm 0.7 \\
(16 \pm 3)\end{array}$ & $\begin{array}{c}0 \pm 1 \\
(-6 \pm 4)\end{array}$ & $\begin{array}{c}0.5 \\
(0.9)\end{array}$ & $\begin{array}{c}0.1 \\
(<0.01)\end{array}$ \\
\hline
\end{tabular}

values of $m_{c}$. As such, there is a closer association of Eq. (1) with the bandwidth of our typical implementations at sites of finescale overprediction than that which typifies the dataset as a whole.
Sheen et al. (2013) similarly argue against an influence of integration limits on the finescale overprediction signal seen in DIMES data. In that study, the High Resolution Profiler 2 (HRP2) measured current velocities that
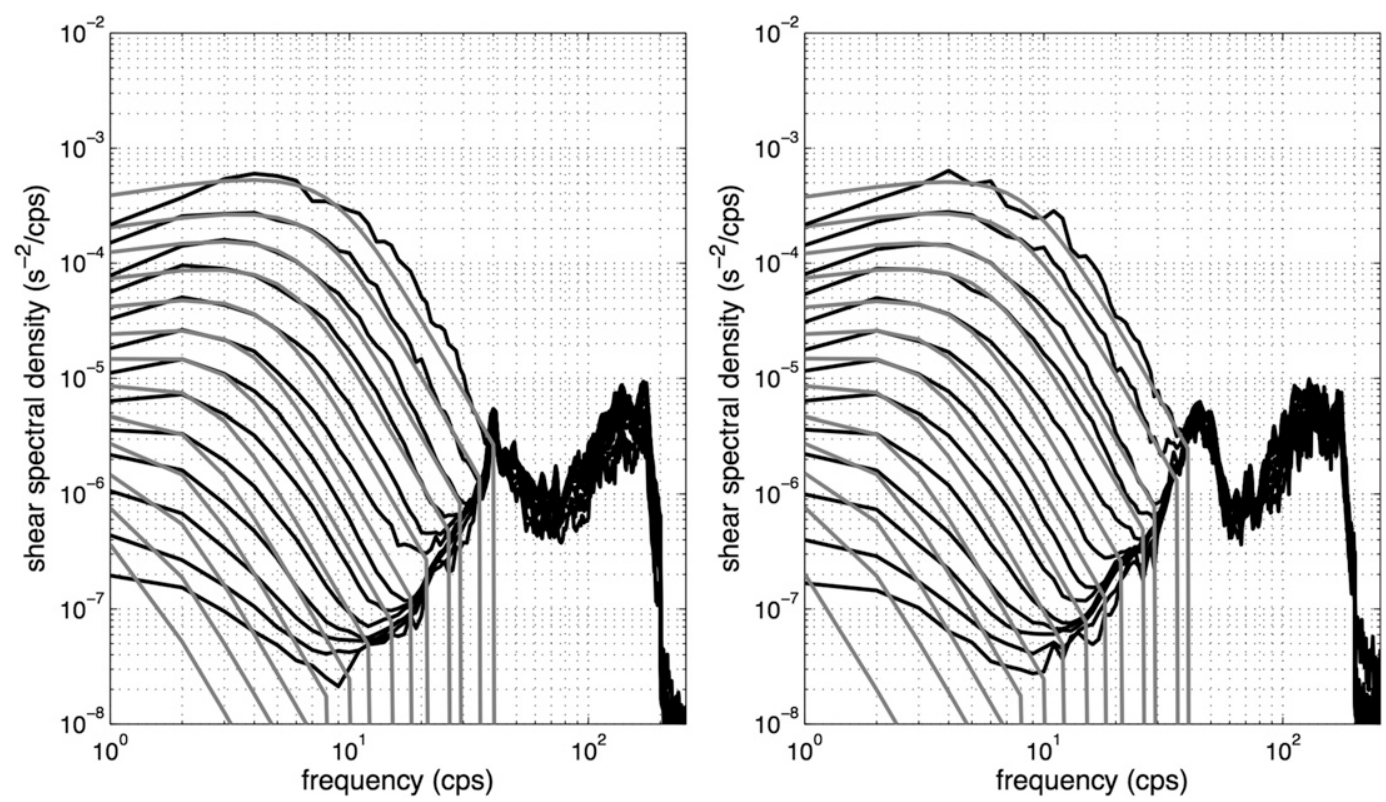

FIG. 6. Averaged microstructure shear spectra from the bottommost $640 \mathrm{~m}$ for the eight special stations. Spectra are binned in terms of the dissipation level in the range from $5 \times 10^{-12}$ to $1 \times 10^{-7} \mathrm{~W} \mathrm{~kg}^{-1}$ in increments of $1 \times$ $10^{-x}, 2 \times 10^{-x}, 5 \times 10^{-x}$, and so on. Gray lines show the universal velocity shear spectrum using an analytic expression found by fitting the Nasmyth data (Oakey 1982). (right) and (left) Spectra from the two independent probes. 


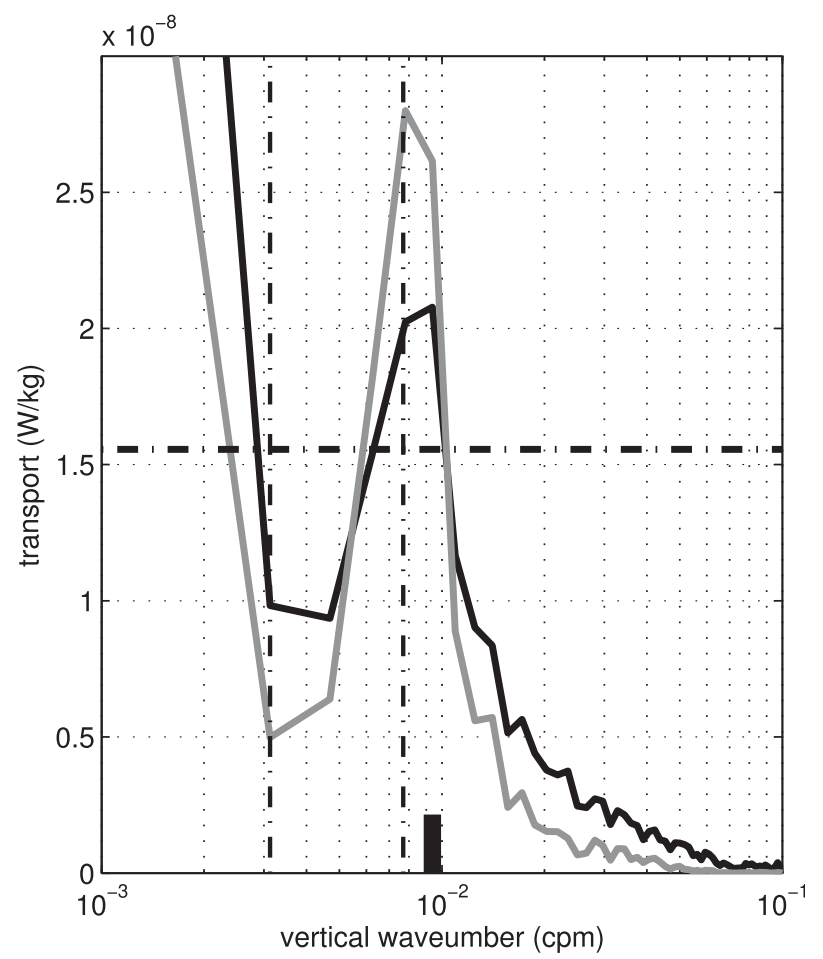

FIG. 7. Estimates of the average spectral energy transport in vertical wavenumber space based on finescale shear and strain estimates in the bottommost transform bin (within $640 \mathrm{~m}$ of the bottom) for the eight special stations. The nominal ray-tracing formula [Eq. (1)] is shown in black, and the local approximation [Eq. (2)] is shown in gray. The dashed black horizontal line uses shear spectral levels averaged to $m_{c}$ and a similarly averaged estimate of the shear-to-strain ratio [the finescale parameterization implementation described in Polzin et al. (1995)]. Here $\left(1+R_{f}\right)$ where $R_{f}$ is a flux Richardson number times the observed dissipation is shown by the height of the thick black line at the average $m_{c}$ vertical wavenumber. The overprediction is represented by the difference in the transport rate given by the height of this line and that of the other various finescale parameterization estimates.

enabled HRP2-deduced shear spectra to be evaluated to $m_{c}$ in most places, and yet these integrations showed the same tendency for overprediction.

In summary, we identify in the SOFine dataset a robust, systematic, and statistically significant tendency for various implementations of the finescale parameterization to overpredict the microstructure-derived dissipation rate near the seafloor in key locations characterized by ACC jet flow and elevated near-bottom dissipation rates. The signal's reproducibility across various parameterization implementations, a lack of atypical instrument noise at these locations, as well as the documentation of a similar phenomenon in DIMES data (Sheen et al. 2013), all suggest that the overprediction we see is not likely to reflect instrumentation and/or parameterization biases. An alternative hypothesis, that finescale overprediction is underpinned by physics relevant to the environment and/or wave field in the locations in which it occurs, is explored in the following section.

\section{b. Relation to physical parameters}

\section{1) RELATION TO PREDiCTORS OF BOTTOM WAVE GENERATION}

The tendency for the finescale parameterization to overpredict close to the bottom, and in particular inside the ACC jets where the near-bottom dissipation rate is high, suggests a possible link to bottom-generated waves. Maps of the locations of near-bottom finescale overprediction with respect to the ACC frontal locations (Fig. 1b) and small-scale topographic roughness (Fig. 1d) give the visual suggestion that overprediction tends to occur inside the ACC jets and/or in regions of rougher topography. Consistently, an analysis of the dependence of the $\epsilon$ ratio on topographic roughness and near-bottom flow speed shows that finescale overprediction is enhanced when these quantities are both large (Fig. 8). This dependence underpins the tendency we see for finescale overprediction to be associated with large values of the theoretically predicted lee-wave energy flux documented in Fig. 9. ${ }^{1}$ The distribution of $\epsilon$ ratio values for stations with a small versus large predicted wave flux (specifically less than or greater than the dataset mean) (Fig. 9b) shows a distinct distribution for each group: the distribution of $\epsilon$ ratio values at locations with a large predicted wave flux has approximately $30 \%$ more observations of $\epsilon$ ratio values greater than one, as well as a larger mean, median, and mode value relative to the distribution for locations with a small predicted wave flux. A rank sum test confirms that for stations with large predicted wave fluxes, the median value of the $\epsilon$ ratio observed is statistically distinct from that for stations with small predicted wave fluxes to the $95 \%$ significance level (with a $P$ value of $<0.01)$. Taken together, Figs. 8 and 9 make a strong suggestion that the finescale overprediction observed tends to be associated with sites where we expect bottom wave generation to be enhanced.

\footnotetext{
${ }^{1}$ The prediction of the lee-wave energy flux considered here is based on the linear theory of Bell (1975) and modified by Nikurashin and Ferrari (2010b) to account for the suppression of energy radiation at super-critical topography [see Nikurashin and Ferrari (2011) for further details]. It is implemented with the SOFine data using the observed near-bottom (i.e., average in the bottommost $500 \mathrm{~m}$ ) flow speed and stratification and the small-scale topographic parameters estimated by Nikurashin and Ferrari (2011). See Waterman et al. (2013) for further details.
} 


\section{2) RELATION TO INTERNAL WAVE FIELD CHARACTERISTICS}

Consistent with the association between enhanced near-bottom dissipation and upward-propagating, less near-inertial waves (Waterman et al. 2013; Sheen et al. 2013), finescale overprediction also displays an association with low values of the shear-to-strain ratio $R_{\omega}$ and a shear polarization suggesting upward internal wave energy propagation (Fig. 10). ${ }^{2}$ Consideration of the $\epsilon$ ratio as a function of wave frequency implied by the $R_{\omega}$ values (Fig. 10a) shows a systematic association of finescale overprediction with less near-inertial values of this approximation to the waves' bulk frequency (i.e., low values of $R_{\omega}$ ). Further, a view of the distribution of $\epsilon$ ratio values for locations having counterclockwise $(\mathrm{CCW})$ shear polarization versus clockwise $(\mathrm{CW})$ shear polarization (Fig. 10b) indicates a systematic association of finescale overprediction with an inferred, upward, internal wave energy flux: at locations with $\mathrm{CW}$ polarization, the finescale parameterization has a statistically significant greater tendency for overprediction with a rank sum test indicating distinct median values of the

\footnotetext{
${ }^{2}$ The shear-to-strain variance ratio $R_{\omega}$ is defined as $R_{\omega}=$ $\left\langle V_{z}^{2}\right\rangle /\left(N^{2}\left\langle\zeta_{z}^{2}\right\rangle\right)$. Under a single wave approximation, it is a measure of the internal wave field's aspect ratio and intrinsic frequency $\omega$ given by $\omega=\sqrt{\left[\left(R_{\omega}+1\right) /\left(R_{\omega}-1\right)\right]} f$, where $f$ is the inertial frequency (Henyey 1991; Hughes and Wilson 1990; Polzin et al. 1995). The polarization ratio $\langle\mathrm{CW}\rangle /\langle\mathrm{CCW}\rangle$ is the ratio of clockwise $(\mathrm{CW})$ - to counterclockwise $(\mathrm{CCW})$-polarized shear variance integrated over the vertical wavenumber band of interest. Its value relative to one indicates a dominance of $\mathrm{CW}$ - to $\mathrm{CCW}$-polarized shear, which can suggest the dominant direction of the energy flux of the sum of internal waves the variance is assumed to represent. A dominance of $\mathrm{CCW}$ polarization $(\langle\mathrm{CW}\rangle /\langle\mathrm{CCW}\rangle<1)$ generally indicates a dominance of downward-directed internal wave energy propagation (in the Southern Hemisphere). Conversely a dominance of $\mathrm{CW}$ polarization $(\langle\mathrm{CW}\rangle /\langle\mathrm{CCW}\rangle>1)$ generally indicates a dominance of upward internal wave energy flux. However, caution is required in making inferences about the energy flux as the rotary decomposition is not a precise division into upward- and downward-propagating waves. For a single wave going up in the Southern Hemisphere, $\langle\mathrm{CW}\rangle /\langle\mathrm{CCW}\rangle=(\omega+f)^{2} /(\omega-f)^{2}$, so $\langle\mathrm{CW}\rangle /\langle\mathrm{CCW}\rangle$ is $\gg 1$ for near-inertial waves, but asymptotes to 1 as $\omega$ approaches $N$. As such, $\langle\mathrm{CW}\rangle /\langle\mathrm{CCW}\rangle$ may not be indicative of the energy flux in a multichromatic wave field. Attention is drawn to the particular case where high-frequency waves with little rotary signature are propagating in the direction opposite to near-inertial waves. Noting that the energy flux is the product of vertical group velocity $\left(\omega^{2}-f^{2}\right) / \omega m$ and energy, in this case one could have a field with $\langle\mathrm{CW}\rangle /\langle\mathrm{CCW}\rangle>1$ and yet a predominant downward energy flux if the high-frequency waves with little rotary signature are propagating downwards opposite to the near-inertial waves propagating upwards. For full details on the calculation of both $R_{\omega}$ and $\langle\mathrm{CW}\rangle /\langle\mathrm{CCW}\rangle$ here, see Waterman et al. (2013).
}

CCW-versus CW-polarized $\epsilon$ ratio distributions to the $95 \%$ significance level (with a $P$ value of 0.09 ). The association of near-bottom finescale overprediction with upward-propagating waves is further suggested by the observation of excess $\mathrm{CW}$-polarized energy seen near the bottom at several of the eight special sites, a feature that is seen also in the average rotary spectra for this special station group (Fig. 11). The peak in CWpolarized energy at $\sim 100$-m vertical wavelength in the special station average spectra is at least double that of the CCW-polarized energy at this vertical scale for the same station group and of both the CW- and CCWpolarized energy at this scale for the average of the nonspecial stations. This association of finescale overprediction with less near-inertial wave frequencies and shear polarization consistent with upward internal wave energy propagation, in particular for the special eight stations, further suggests a link between finescale overprediction and bottom-generated waves of lee-wave or tidal origin.

The excess CW-polarized energy seen near the bottom at stations where the near-bottom finescale overprediction is particularly large is also noteworthy because it is seen exclusively at relatively large $[O(100 \mathrm{~m})]$ vertical scales and as relatively narrowband signals. Individual near-bottom rotary spectra (not shown) show narrow peaks with amplitudes up to an order of magnitude larger than the GM shear variance level at vertical wavelengths $\lambda_{z}$, ranging from $\sim 20$ to $\sim 200 \mathrm{~m}$, while the special station average shows enhanced $\mathrm{CW}$-polarized energy in two distinct narrowband peaks centered at $\lambda_{z}=106 \mathrm{~m}$ and $\lambda_{z}=58 \mathrm{~m}$, respectively (Fig. 11). Similar signatures are seen in the spectral shape of near-bottom shear and strain for these stations (Fig. 12): individual stations show narrowband peaks in shear and strain variance at intermediate vertical scales in the range of 20-200 m (not shown), while the special station averages show enhanced variance over a slightly broader range centered at $\lambda_{z}=106 \mathrm{~m}$. Sheen et al. (2013) similarly document enhanced spectral energy in strain at wavelengths of $O(100 \mathrm{~m})$ at finescale overprediction sites. The peaked spectral shape in the near-bottom spectra at the special sites suggests that the parameterization's tendency to overpredict the dissipation rate at these locations may derive from a failure to satisfy the condition of a flat spectral shape at intermediate vertical scales, as is assumed in the derivation of Eqs. (2) and (3). However, the existence of the overprediction signal also in the finescale parameterization implementation described by Eq. (1) (see Fig. 7), which does not make the flat spectral shape assumption, suggests that this is not the overprediction signal's underlying cause. 


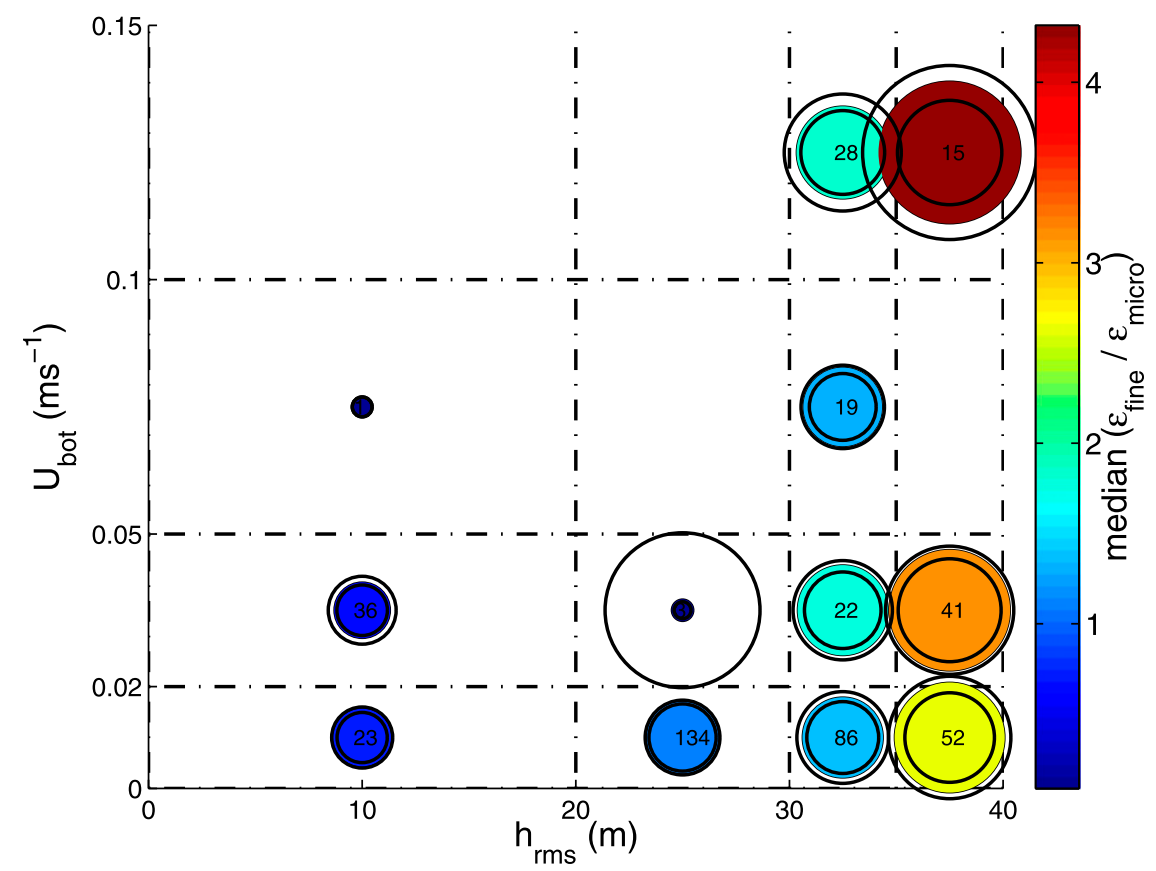

FIG. 8. Bin average median values of the $\epsilon$ ratio as a function of topographic roughness $h_{\text {rms }}$ and the near-bottom background flow speed (average LADCP speed in the bottommost $500 \mathrm{~m}$ ) $U_{\text {bot }}$. Both the size and color of the dot display the median value of the $\epsilon$ ratio. Black circles indicate the $90 \%$ confidence intervals on the median ratio computed via bootstrap sampling. The number inside the circle indicates the number of estimates in each bin.

\section{3) RELATION TO BACKGROUND FLOW CHARACTERISTICS}

Finally, in addition to showing an association with physical parameters key to wave generation and an internal wave field of a particular character, near-bottom finescale overprediction is also found to have a systematic dependence on certain large-scale, that is, background, flow characteristics.

First, finescale overprediction shows a systematic association with the magnitude of the near-bottom background flow shear $\bar{U}_{z}$ (Fig. 13). This relationship is potentially significant, as the importance of wavemean flow interactions relative to nonlinear wavewave interactions in the spectral energy transport in vertical wavenumber space can be gauged by the magnitude of the Froude number $\mathrm{Fr}=\bar{U}_{z} / N$. Here, $\bar{U}_{z}$ is the vertical shear of the subinertial flow and is estimated here from the vertical profile data smoothed by a sliding polynomial fit over a vertical scale of $300 \mathrm{~m}$. A tendency for finescale overprediction to be associated with Froude numbers $O(0.1)$ and greater, a positive linear trend of the $\epsilon$ ratio on Fr, and a statistically significant correlation between the $\epsilon$ ratio and $\mathrm{Fr}$, all suggest that wave-mean flow interactions may play a role in underpinning the observed finescale overprediction here. This suggestion is further endorsed by a closer look at the character of the large-scale background flow at the special sites (Fig. 14a). The typical magnitude of these background flow shears (on the order of $\Delta U \sim 0.1 \mathrm{~m} \mathrm{~s}^{-1}$ in $\Delta z \sim 1000 \mathrm{~m}$ ) results in Froude numbers of order 0.1, given the near-bottom background $N$ on the order of $10^{-3} \mathrm{~s}^{-1}$. This order of magnitude Froude number is large enough for wavemean flow interactions to be playing a significant role in the wave dynamics (Polzin et al. 2014). A similar association of finescale overprediction with Froude number documented for the DIMES data (Sheen et al. 2013) also provides support for this hypothesis.

In addition to being characterized by large magnitudes of the near-bottom background flow shear, Fig. 14a also reveals that all of the special sites that show atypically large near-bottom finescale overprediction also exhibit an atypical negative-signed background shear (background flow magnitude decreasing with height above bottom) in approximately the bottommost $1000 \mathrm{~m}$. This observation is potentially very significant as a background shear of this sign will force the evolution of the properties of an upward-propagating wave oriented into the background flow (as is the case with lee 


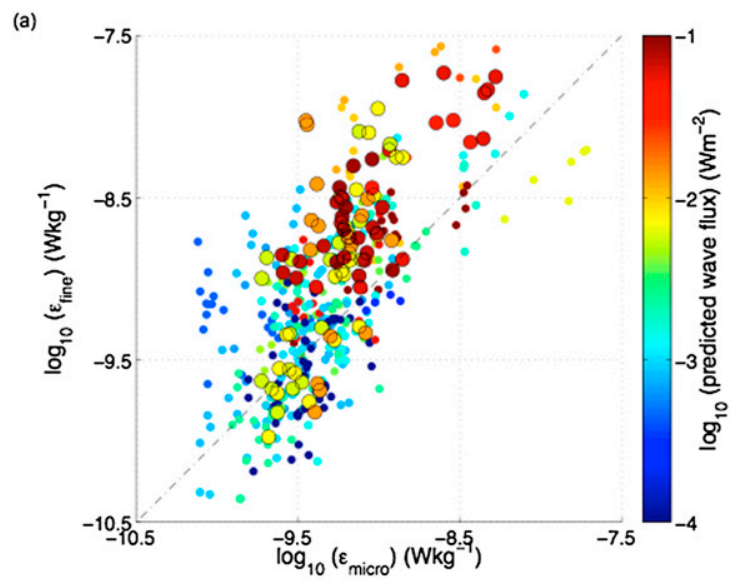

(b)

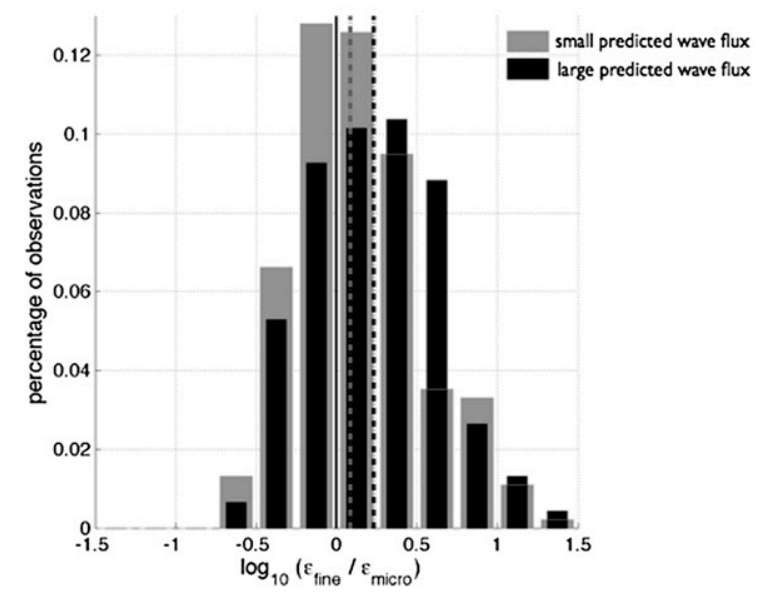

FIG. 9. (a) Scatterplot of $\log _{10}\left(\epsilon_{\text {micro }}\right)$ versus $\log _{10}\left(\epsilon_{\text {fine }}\right)$ colored by the local predicted lee-wave energy flux (calculation described in the text). Each point corresponds to one depth bin for which the $\epsilon_{\text {fine }}$ calculation was made, and all depth bins centered at or below $500-\mathrm{m}$ depth are included. The special eight stations are shown by larger symbols outlined in black. (b) The distribution of $\epsilon$ ratio values for stations with a small predicted wave flux (less than the dataset mean value of $2.4 \mathrm{~mW} \mathrm{~m}^{-2}$; gray) versus stations with a large predicted wave flux (greater than the dataset mean value of $2.4 \mathrm{~mW} \mathrm{~m}^{-2}$; black). Dashed-dotted lines show the median values for each group ( $\epsilon$ ratio values of 1.2 and 1.7, respectively).

waves) toward those associated with an internal wave critical layer situation. In such a scenario, we expect wave-mean flow interactions to transfer wave energy to smaller vertical scales, affecting an evolution of the wave frequency toward the inertial frequency and the wave's vertical group velocity toward zero. Consistent with this, the near-bottom vertical profiles of various wave and turbulent properties at these sites (Fig. 14) show, in some cases, signatures suggestive of this evolution. These signatures, and the hypothesis of wavemean flow interactions in this evolution as being key to the finescale overprediction observed, are considered in the following discussion.

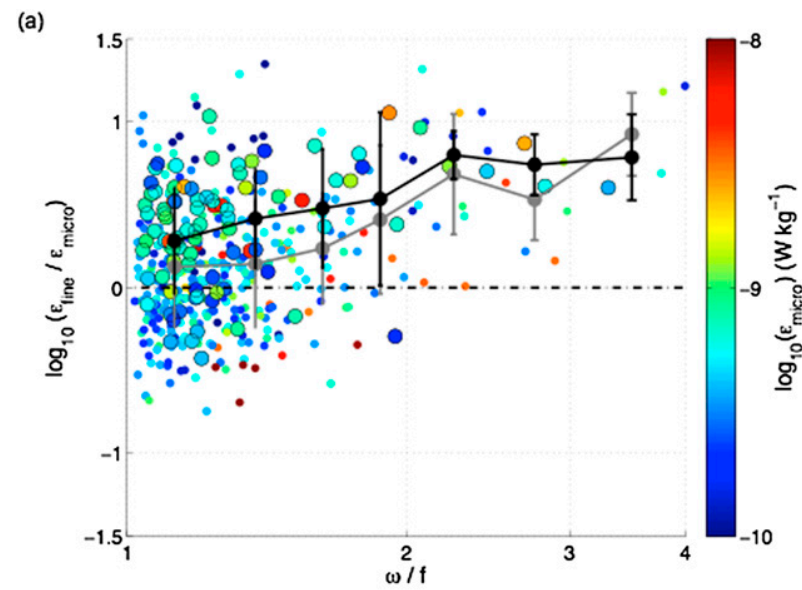

(b)

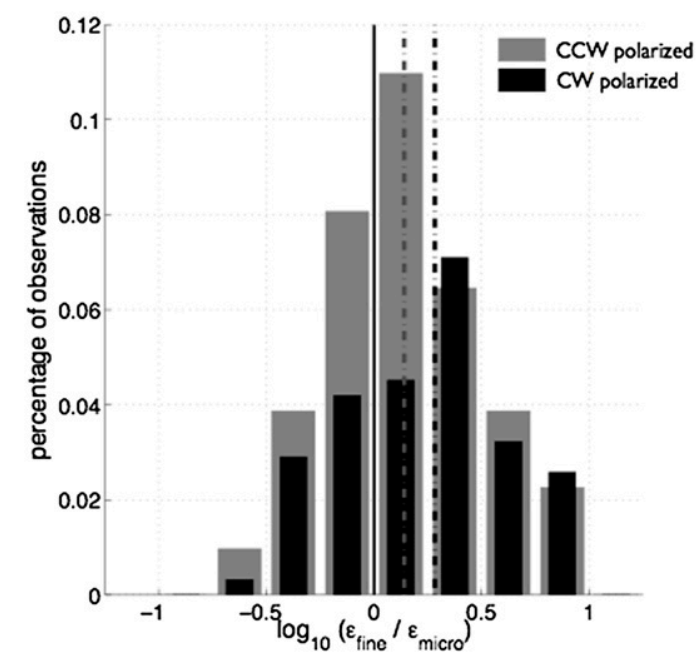

FIG. 10. The dependence of the $\epsilon$ ratio on (a) wave bulk frequency diagnosed from the shear-to-strain variance ratio $R_{\omega}$ and (b) shear polarization. In (a), points are colored by the depth bin average value of $\epsilon_{\text {micro, }}$, and bin average values of the $\epsilon$ ratio are shown in gray for all data and black for data from the special eight stations only. Error bars show the std dev in the bin average mean. In (b), the distribution of $\epsilon$ ratio values for locations with a predominance of CCW-polarized shear is shown in gray and for locations with a predominance of $\mathrm{CW}$-polarized shear in black. Only locations with a distinct polarization $\left[\left|\log _{10}(\mathrm{CCW} / \mathrm{CW})\right| \geq 0.25\right]$ are included. Dashed-dotted lines show the median values for each group ( $\epsilon$ ratio values of 1.4 and 1.9 , respectively).

\section{Discussion}

The purpose of this paper is to document an unanticipated, robust, and systematic overprediction of the turbulent dissipation rate predicted by various implementations of the finescale parameterization relative to microstructure observations in the bottommost $1500 \mathrm{~m}$ at special locations. These sites are typically characterized by large predictions of lee-wave energy flux (i.e., large near-bottom flow speeds and moderate topographic 


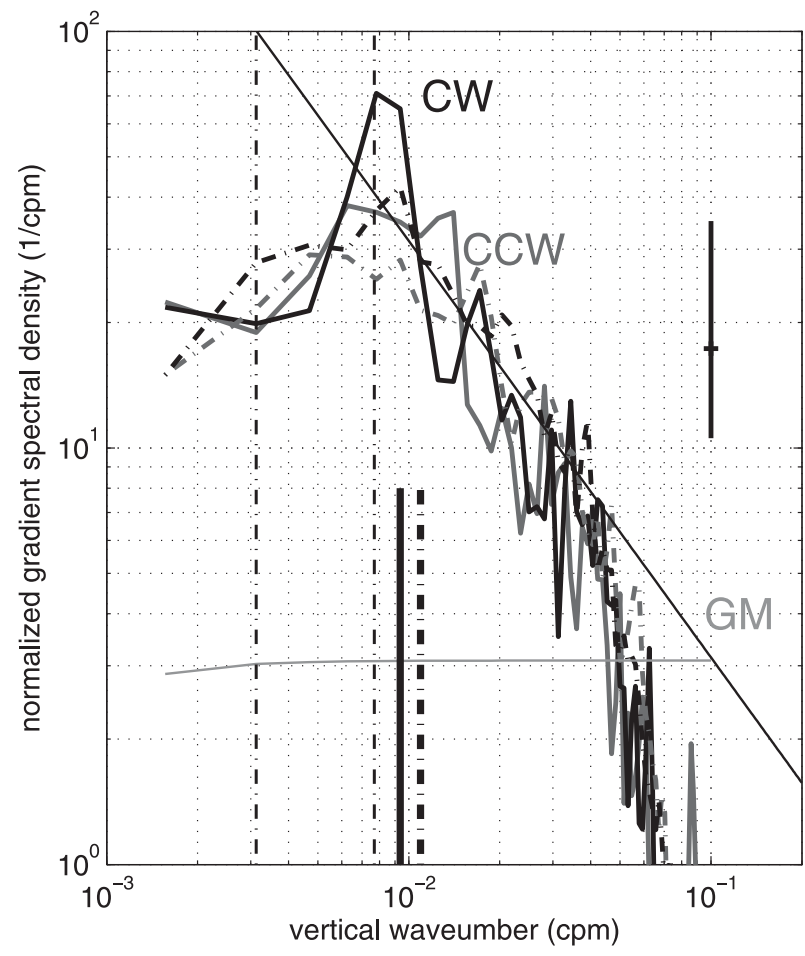

FIG. 11. The special station average $\mathrm{CW}$-polarized buoyancy frequency-normalized shear spectra in the bottommost transform bin (corresponding to within $640 \mathrm{~m}$ of the bottom; thick black solid line). For comparison, the corresponding average $\mathrm{CCW}$-polarized spectra (thick gray solid line), the average $\mathrm{CW}$ - and $\mathrm{CCW}$-polarized shear spectra for all other stations (thick black and gray dasheddotted lines, respectively), the GM76 shear spectral model/2 appropriate for the mean near-bottom stratification of the eight special stations (thin light gray line), and the saturation spectra assuming a value of $m_{c}=1 / 100$ cycles per meter (cpm) (thin black line) are also shown. The thick black vertical line at a vertical wavelength of $106 \mathrm{~m}$ indicates the average value of $m_{c}$ computed from the shear variance level of the average shear spectrum for the special stations. The thick dashed-dotted vertical line at a vertical wavelength of $91 \mathrm{~m}$ indicates the average value of $m_{c}$ for all other stations. The confidence interval indicates the $95 \%$ confidence interval assuming 16 degrees of freedom.

roughness); low shear-to-strain ratio values (suggesting less-than-typical near-inertial wave frequency content); rotary spectra that indicate a predominance of an upward internal wave energy flux; shear and strain spectra that show enhanced relatively narrowband variance at vertical wavelengths on the order of $100 \mathrm{~m}$; relatively large Froude numbers based on the near-bottom shear of the background flow; and a background flow with a systematic backing tendency. The overprediction signal's systematic association with these physical parameters, in combination with its reproducibility across various parameterization implementations, a lack of association with various metrics of instrumental noise, and the documentation of a similar signal with similar

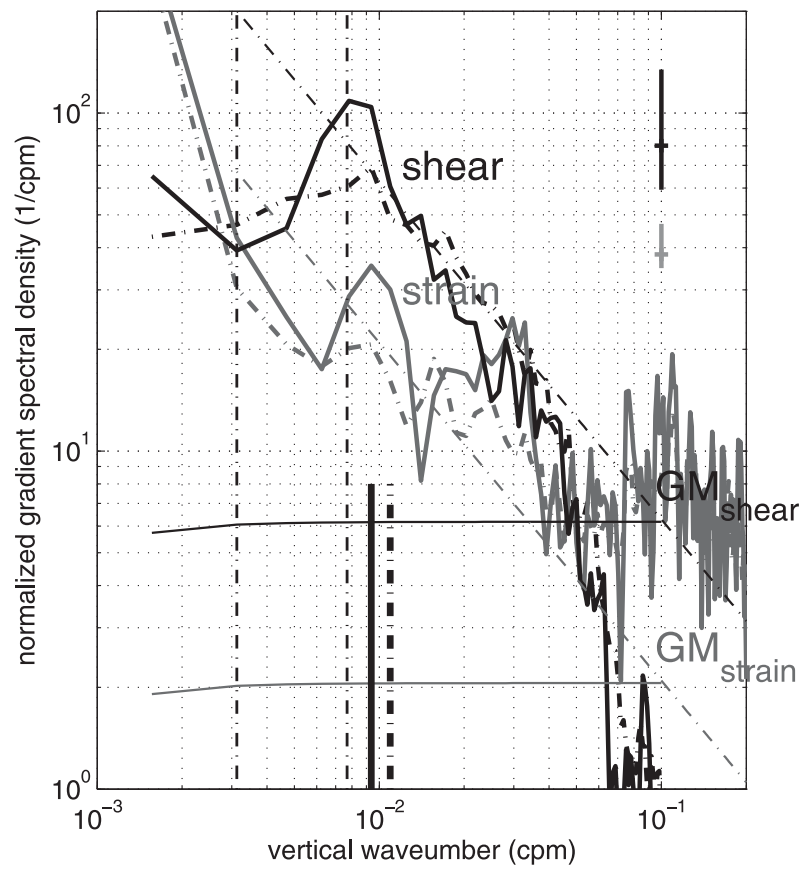

FIG. 12. Equivalent special station average spectra as in Fig. 11, but for buoyancy frequency-normalized shear (thick black line) and strain (thick gray line). These averages for all other stations are shown by the thick dashed-dotted lines. The GM76 model shear and strain spectra (thin solid black and gray lines) and the saturation spectra assuming a value of $m_{c}=1 / 100 \mathrm{cpm}$ (thin dasheddotted black and gray lines) are also indicated. The thick black solid and dashed-dotted back vertical lines indicate the average value of $m_{c}$ as in Fig. 10. The confidence intervals indicate the $95 \%$ confidence interval assuming 16 degrees of freedom.

dependencies on environmental and wave field characteristics in Sheen et al. (2013), provides strong support for a physical interpretation of the phenomenon. However, the explanation for what underpins the tendency for the finescale overprediction at these sites remains an open question. There are several plausible explanations, and we speculate on leading candidates in the following discussion. Although the data available do not permit a definitive test of the various hypotheses, they do provide useful clues to judge the feasibility of various proposals and to motivate future studies.

\section{a. The implications of a nonequilibrated wave field close to generation sites}

As discussed in section 2, the finescale parameterization rendered in Eq. (1) has roots in ray-tracing simulations summarized in Henyey et al. (1986). These simulations assume a background wave field consistent with the spatially homogeneous, vertically isotropic GM76 spectrum. As such, there exist open questions regarding the applicability of the parameterization close to sites of strong internal wave generation. Here, we 


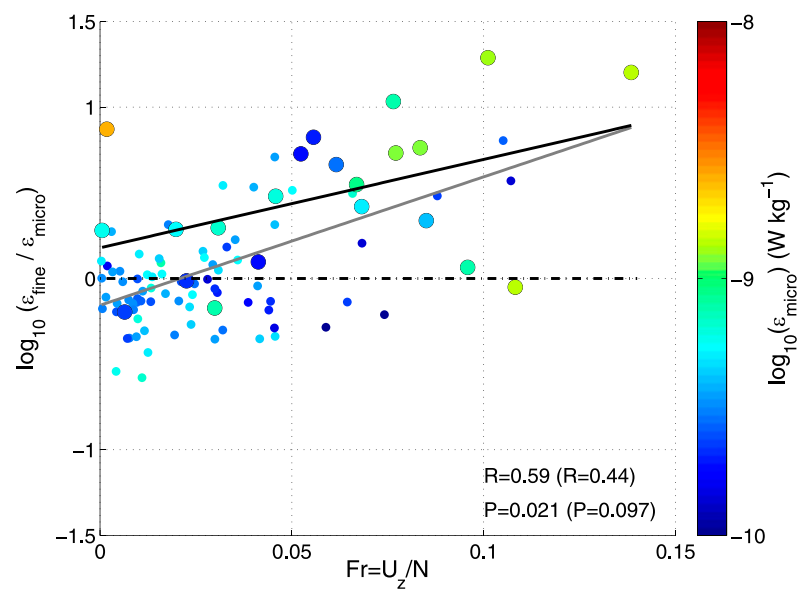

FIG. 13. The relation between the Froude number, based on the vertical shear of the background flow and as defined in the text, and finescale overprediction near the bottom (specifically at heights of $1000 \mathrm{~m}$ or less). Points are colored by the value of $\epsilon_{\text {micro. }}$. Lines show the result of a linear regression of the $\epsilon$ ratio on Froude number for the full dataset (black) and the special eight stations only (gray). Regression statistics are displayed in the lower right-hand corner for the full dataset with those corresponding to the calculation using data from the special eight stations only in parentheses.

expect excess energy at a preferred vertical scale dictated by the bathymetry and the incident current and as such a violation of the assumptions of spatial homogeneity and vertical isotropy.

It should be noted that departures from the spatially homogeneous and vertically isotropic background state are handled in the finescale parameterization by assuming that the background/test wave correlations depend only on the background vertical shear variance.
Given this assumption, downscale transports are proportional to the expected value of the test wave aspect ratio (Polzin et al. 1995). This dependence manifests itself in the functional dependence on wave frequency $h\left(R_{\omega}\right)$ in the finescale parameterization rendered in Eq. (3). This frequency-corrected form of the parameterization has proven successful in predicting the dissipation rates consistent to within a factor of 2 for a number of wave fields exhibiting non-GM characteristics; see Polzin et al. (1995) for various examples. The parameterization has also been developed as a successful prognostic tool to parameterize tidal mixing in a decidedly inhomogeneous and anisotropic environment [e.g., Polzin 2004, 2009; see also Fig. 11 of Mauritzen et al. (2002)].

Vertical anisotropy could nevertheless be a key ingredient to explain the observed overprediction here, and an interesting possibility consistent with the observed signals concerns the behavior of high-frequency waves in a vertically anisotropic near-inertial wave field. The condition for resonant interaction of internal waves is that the three wavenumbers and frequencies sum to zero. For two high-frequency waves $\left(\omega\right.$ and $\left.\omega_{2}\right)$ and a near-inertial wave $\left(\omega_{1} \cong f\right)$, the condition on the frequencies becomes $\omega-\omega_{1}-\omega_{2}=0$. With $\omega$ and $\omega_{2} \gg \omega_{1}$, a Taylor series expansion provides $m_{1}(\partial \omega / \partial m) \cong f$, that is, the approximate resonance condition that the vertical group velocity of the high-frequency wave matches the vertical phase speed of the near-inertial wave. Because the vertical phase speed and group velocity of internal waves are in opposite directions, one might anticipate a tendency of the finescale parameterization to overestimate the observed dissipation if all waves are propagating in the same direction. The plausibility of this (a)

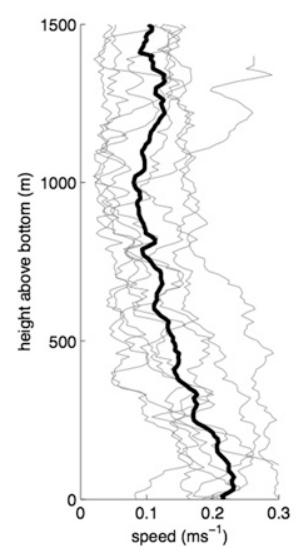

(b)

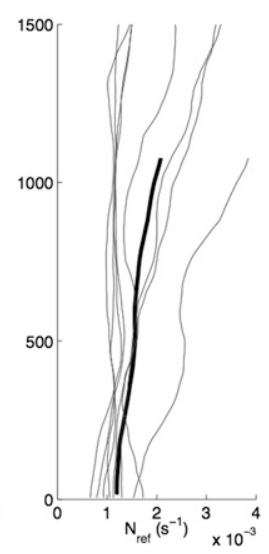

(c)

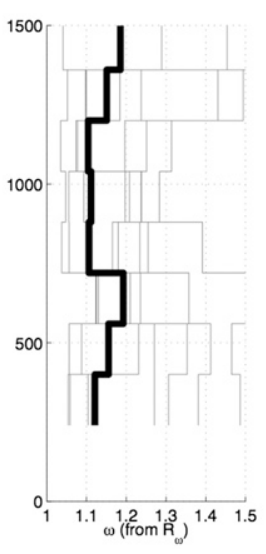

(d)

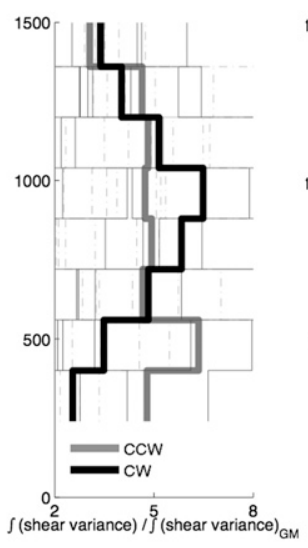

(e)

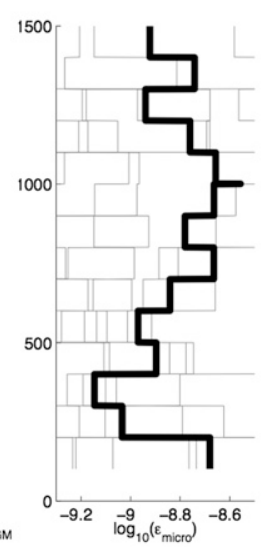

(f)

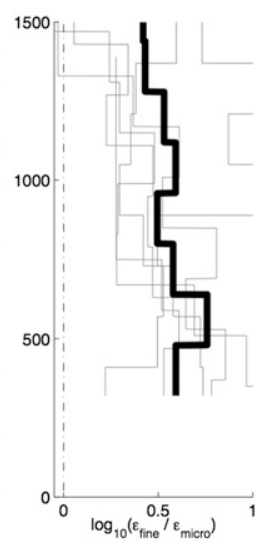

FIG. 14. Vertical profiles as a function of height above the bottom of (a) speed; (b) background stratification $N_{\text {ref }}$; (c) wave intrinsic frequency $\omega$, inferred from the shear-to-strain variance ratio $R_{\omega}$; (d) CCW- (gray) and CW-polarized (black) shear variance integrated over integration limits 1 and normalized by the equivalent GM shear variance level; (e) $\epsilon_{\text {micro; }}$; and (f) the $\epsilon$ ratio for the eight special stations. The black line is the composite mean for all stations. 
hypothesis is the subject of ongoing work. Moored data do document a tendency of near-inertial waves to enter into such critical layer situations in combination with high-frequency waves exiting them (Polzin 2010), and Brearley et al. (2013) report that mooring measurements to the east of Drake Passage show CW polarization in association with near-inertial waves, but that total near-bottom shear variance is dominated by higherfrequency waves. Further, although vertical anisotropy characterizes both the tidal and lee-wave generation problem, in the former $m_{c} \cong N / U_{\mathrm{BT}}$, whereas in this leewave example $m_{c} \cong 10 N / U_{g}$ (here $U_{\mathrm{BT}}$ and $U_{g}$ are the amplitudes of the barotropic tidal velocity and geostrophic flow velocity, respectively). This makes the leewave example more linear and potentially more prone to the resonant criteria described above. The finescale parameterization lacks a first principles derivation, and until this derivation is accomplished it is difficult to assess whether the formulas are being applied outside their domain of applicability in these cases.

\section{b. Short-circuiting of the downscale energy cascade by boundary conditions}

The central assumption of the finescale parameterization, that is, that the downscale energy transport toward the scales of turbulent production is driven by nonlinearity in the internal wave field, may be violated by boundary conditions. As discussed in more detail in Polzin et al. (2014), the nonlinear downscale energy cascade past $m=m_{c}$ may be short-circuited by internal wave scattering (Muller and Xu 1992) and reflection (Eriksen 1985) at a boundary, resulting in the insertion of significant energy at vertical scales smaller than $m_{c}^{-1}$. In this instance, failure of the finescale parameterization should be anticipated. This phenomenon plausibly explains the poor agreement between finescale parameterization estimates and microstructure observations documented by Kunze et al. (2002), where estimates of the transition wavelength $\lambda_{c}=2 \pi / m_{c}$ in energetic cases exceeded the water depth.

We argue that this phenomenon is unlikely, however, to be the explanation for the discrepancy observed here. First, estimates of $\lambda_{c}$ that characterize the energetic sites prone to finescale overprediction are relatively small, averaging $O(100 \mathrm{~m})$ in the bottommost transform bins at the special sites. These $\lambda_{c}$ values imply that the boundary layer influence will extend up to a distance of $(1 / 2) \lambda_{c}=O(10 \mathrm{~m})$ into the interior before being dissipated by overturning or instabilities. In contrast, the near-bottom overprediction signal extends over a distance $O(1000 \mathrm{~m})$. Further, the overprediction signal is robust when the parameterization is computed using transform intervals stepped off the bottom well above what we anticipate is sufficient to be far enough from forcing and boundaries to evoke a cascade representation.

The failure of the parameterization should also be anticipated in boundary layers where dissipation is associated with viscous stresses. However, such a shortcircuit of the nonlinear process by boundary conditions implies a systematic parameterization underprediction, rather than the overprediction we document.

\section{c. An influence of wave-mean flow interactions}

A second key difference between sites of tidal and leewave generation is the presence of a mean shear, and the tendencies for finescale overprediction to be associated with relatively large background flow shears and correlated with the implied Froude numbers suggest, as discussed in section $3 \mathrm{~b}$, the possibility of wave-mean flow interactions playing a significant role in wave evolution. Further, the presence of a backing flow (background flow magnitude decreasing with height) at all of the special sites suggests the potential for waves oriented into the shear (as is the case for lee waves) to evolve toward critical layer wave characteristics. Near-bottom vertical profiles of various wave and turbulent properties at these sites (Fig. 14) show, in some cases, signatures suggestive of this evolution: vertical profiles of $R_{\omega}$ at most of the special stations show large, vertically localized maxima at heights ranging from 500 to $1250 \mathrm{~m}$ (consistent with the expected evolution of the wave frequency toward the inertial frequency), and on average, the upward-propagating (i.e., $\mathrm{CW}$ polarized) wave energy is seen to increase with height above bottom to $\sim 1000 \mathrm{~m}$ (the height of a local maximum in the average dissipation profile and consistent with the expected increase in the upward-propagating wave amplitude to the average height of the change in sign of the background shear). In contrast, downward-propagating (i.e., CCW polarized) energy shows no systematic vertical structure in this range of heights, although the signature of excess $\mathrm{CCW}$ variance in the bottommost $\sim 500 \mathrm{~m}$ is intriguing and may indicate the relevance of resonant interactions discussed above. Finally, an exploration of the "what if" scenario to evaluate whether the scales of the typical vertical scale and inferred wave frequency we observe at the off-bottom height are consistent with the expected evolution of a lee wave in the observed shear (see appendix B) suggests that the observed scales are order of magnitude consistent with this scenario. Taken together, these observations support the suggestion that wave-mean flow interactions, and more specifically the evolution of bottom-generated waves toward critical layer situations, play a key role in the evolution of the waves in question at the sites prone to large finescale overprediction. 
The implications of these wave-mean flow interactions in modifying the physics described by the finescale parameterization are not straightforward. In general, a critical layer scenario implies the transport of energy to smaller vertical scales with some fraction of the wave energy being lost to work against the mean before wave breaking. This lost fraction of wave energy may underpin the parameterization's tendency to overpredict in these cases. However, this description of the wave evolution is one that is wave frequency dependent, and because the lee-wave field generated by a continuous topographic spectrum has a continuous intrinsic frequency spectrum, we expect a series of critical levels, one for each intrinsic frequency. Further, because the background velocity profile does not back to very small values in this particular case, only the lowest-frequency waves will be trapped at their respective critical level while higher-frequency waves will be permitted to escape. The evolution of wave properties toward their critical level characteristics will, for many wave frequencies, be partial, with the tendencies reversing sign above the height where the background flow magnitude starts to increase. The details of the influence of these interactions on the vertical profiles of wave and turbulent properties and the expected mismatch between finescale parameterization predictions and the actual dissipation rate are a topic of future study.

\section{d. The role of horizontal wave propagation and advection}

A nonlocal dissipation of the radiated energy owing to the waves' horizontal and vertical propagation and the horizontal advection by the generating flow was proposed in the introduction as a plausible explanation for the mismatch between the theoretical prediction for power input by lee-wave generation and the rate of observed near-bottom turbulent dissipation. If we again assume a lee-wave characterization for the near-bottom waves at the special sites, the observed scales of the vertical wavenumber and wave frequency at $1000-\mathrm{m}$ height can be used to arrive at scale estimates for the horizontal and vertical components of the lee-wave group velocity both at the bottom and at 1000-m height (see appendix B for details). This thought experiment yields scale estimates for the wave's vertical propagation speed that is an order of magnitude less than its horizontal propagation speed and two orders of magnitude less than the advection speed of the generating flow. These scale estimates thus provide quantitative support for the claim that horizontal wave propagation and background flow advection will play a significant role in the evolution of a lee wave between its generation at the bottom and its arrival at the height where the background shear changes sign and its evolution toward critical layer properties is halted. Further, they suggest that considering one-dimensional vertical profiles of wave and turbulent properties as records of the wave's evolution as it propagates upward from the bottom relies on the statistical homogeneity of the leewave generation process.

\section{e. Final remarks}

In closing, the title of our paper claims that a "suppression of internal wave breaking" underpins the nearbottom finescale parameterization overprediction we have documented here and in DIMES observations. It is important to recognize, however, that, given the limitations of these datasets, we cannot definitively claim that a suppression of wave breaking is the root cause of the overprediction signal. Testing this hypothesis is also the subject of ongoing work. Nevertheless, this interpretation is useful as the overprediction can be considered as an effective suppression of internal wave breaking in widespread instances where finescale parameterization results are interpreted as a measure of the internal wave-driven turbulent mixing rates (e.g., Naveira Garabato et al. 2004; Kunze et al. 2006; Wu et al. 2011). The lack of account of this "effective suppression" may contribute in part to relatively large turbulent dissipation and mixing rates predicted in the Southern Ocean in regions of rough topography by these finestructure-based studies.

It is prudent to consider the implications of these results for the utility of the finescale parameterization to obtain the subbasin- to global-scale estimates of diapycnal diffusivity that is in demand by both the observational and numerical modeling communities. Is the parameterization underperforming its specifications? Is there a need to modify it or reevaluate past finescale parameterization mixing estimates? In response to these questions, we first note that the SOFine observations show that, in general, all the various implementations of the finescale parameterization that we consider yield a good prediction of the microstructure-derived dissipation rate. The overprediction we document is a feature only of specific locations that appear in association with specific conditions. As a consequence, the bias we identify does not have a global implication. Instead it applies where the relevant physics is likely to include additional processes to those represented by the finescale parameterization, that is, where the downscale cascade effected by wave-wave interactions may not be the whole story.

In line with this description, we do not view these results as exposing intrinsic deficiencies of the parameterization or as a call for finescale parameterization modification. The finescale parameterization describes the dynamics of wave-wave interactions that lead to 
a downscale cascade to the wave-breaking scale. The results presented here do not suggest that the physics of this process are poorly represented. Instead we interpret these results as a call for the incorporation of additional physics (potentially wave-mean flow interactions) and an understanding of how closures for a finite amplitude and likely strongly nonlinear parameter regime evolve from the resonant characterization. A suggested way forward is to work to identify and represent the impact of these new process(es) alongside those represented by the finescale parameterization and in the meantime to continue to usefully apply the finescale parameterization with awareness of the causes of potential bias and careful implementation.

Acknowledgments. The SOFine project is funded by the United Kingdom's Natural Environmental Research Council (NERC) (Grant NE/G001510/1). The VMP was also purchased with this support. We thankfully acknowledge the support of Rockland Scientific International, especially for their timely advice delivered during the SOFine expedition. We are grateful to all the scientists, technicians, officers, and crew of the RRS James Cook for their hard work in making the SOFine cruise a very successful one. SW acknowledges the support of an ARC Discovery Early Career Research Award (Grant DE120102927), as well as the Grantham Institute for Climate Change, Imperial College London, and the ARC Centre of Excellence for Climate System Science (Grant CE110001028). ACNG acknowledges the support of a NERC Advanced Research Fellowship (Grant NE/C517633/1). KLP acknowledges support from Woods Hole Oceanographic Institution bridge support funds. We thank Eric Kunze for useful discussions and five anonymous reviewers of an earlier manuscript for their suggestions on how to explore the overprediction signal in more detail.

\section{APPENDIX A}

\section{LADCP Noise Characteristics and Their Relation to the Finescale Parameterization Overprediction Observed}

Bias in finescale parameterization estimates may be introduced by deficiencies in their implementation and by the failure to recognize instrumental artefacts. Potentially the most notable instrument-related source of bias is the introduction of spurious signals to LADCP velocity finestructure observations by measurement noise and processing procedures. This may cause considerable (by up to an order of magnitude) overestimation of $\epsilon$ if not identified and excluded from the shear variance calculation implicit in the parameterization (Polzin et al. 2014). In the present study, the issues of LADCP noise and the impact of processing procedures challenges the ability to attribute discrepancies between estimates of $\epsilon$ derived from microstructure versus those predicted by the finescale parameterization to new physics.

The purpose of this appendix is to look in detail at the LADCP noise characteristics associated with the LADCP measurements in this particular study and to examine their relation to the finescale parameterization overprediction we document. It will be shown that there is no significant indication of atypical instrumental noise issues at the locations of enhanced mismatch that we discuss. Instead, it will be seen that locations of enhanced $\epsilon_{\text {micro }}-\epsilon_{\text {fine }}$ discrepancy tend to be associated with lower LADCP noise levels than those that characterize the dataset as a whole. This analysis provides support for the claim that it is additional physics not encapsulated by the finescale parameterization, and not systematic bias in the LADCP measurements due to instrument noise, that plays the dominant role in the near-bottom overprediction we document.

We performed various analyses to characterize the LADCP noise characteristics and their relation to the finescale overprediction observed that included the following:

(i) a characterization of a range of diagnostics of LADCP noise considering their distributions in space relative to that of the $\epsilon$ ratio and their average structure with respect to height above the seafloor. The eight special stations showing anomalously high near-bottom overprediction were examined for anomalous signatures in LADCP noise characteristics;

(ii) an examination for systematic dependencies of the $\epsilon$ ratio on LADCP noise diagnostics;

(iii) an inspection of shear and rotary spectra as a function of LADCP noise level; and

(iv) an inspection of shear and rotary spectra as a function of the tendency for the finescale parameterization to over- or underpredict the microstructure-derived dissipation rate.

Noise diagnostics considered included 1) a nominal noise level expressing the statistical uncertainty of the LADCP velocity measurement in optimal conditions. This uncertainty is due to the intrinsic limitations of Doppler sonar systems and is based on the theoretical single-ping accuracy of the system and the number of pings that are averaged per bin $N_{\text {vel }}=\theta / \sqrt{n}$. Here $\theta$ is the theoretical single-ping accuracy taken to be $3.2 \mathrm{~cm} \mathrm{~s}^{-1}$, and $n$ is the number of pings per depth bin of $60 \sim 1000$ in the present study. Also included are 2) characteristics of the 
implied energy spectrum of the LADCP noise $E_{n}(m)=$ $(1 / 2)\left[2 \theta^{2} \operatorname{sinc}^{6}\left(m \Delta_{r}\right) \operatorname{sinc}^{2}\left(m \Delta_{g}\right)\right] /(n \times \mathrm{BW})$. Here $\Delta_{r}$ is the finite range gate of the received signal, $\Delta_{g}$ is the depth grid spacing, and $\mathrm{BW}$ is the bandwidth equal to the Nyquist wavenumber of the averaging interval, that is, a function of the depth grid resolution; see Polzin et al. (2014) for an in-depth discussion. Other diagnostics considered were 3 ) the shear variance per bin as output by the LADCP shear processing method; 4) range estimates of the LADCP that vary according to the scattering levels in the local environment; and 5) various estimates of the LADCP velocity error as output by the LADCP velocity-inversion-processing method. The potential dependence of the overprediction on stratification, characterized by the buoyancy frequency $N$, was also considered, owing to concerns that instrument noise may be enhanced in weakly stratified environments.

Select results of these analyses are shown in Figs. A1A4. From these results we make the following observations:

(i) Vertical profiles of all noise diagnostics show a systematic enhancement of LADCP noise (e.g., an increased nominal noise level, increased shear variance per bin, and decreased LADCP range), as well as a systematic reduction in stratification on average, as you approach the bottom. Sections of these quantities also show some spatial structure, indicating enhanced noise levels at depth along the northern transects (specifically stations 16-19) as well as in deep waters at the northern edges of the central and eastern transects (specifically stations 55-59 and 25-27). However, with the exception of station 19 on the northern transect, these sections do not indicate a tendency for anomalously high noise levels in the locations where we observe the largest near-bottom finescale overpredictions (Fig. A1). Further, the vertical profiles of these diagnostics for the special eight sites do not show signs of enhanced noise levels relative to the overall mean. The average near-bottom nominal noise level and per bin shear variance are in fact lower for the special eight stations relative to the overall mean, while the average near-bottom LADCP range and $N$ value are larger than the overall average. This suggests that the average near-bottom noise level characterizing the special eight stations is actually lower than the overall mean level. We also note that everywhere $N$ exceeds the value of $N_{\text {err }}=4.5 \times 10^{-4} \mathrm{rad} \mathrm{s}^{-1}$ identified by Kunze et al. (2006) as the minimum $N$ for which shear estimates were usable owing to acceptable noise levels.

(ii) A visualization of the potential influence of noise diagnostics on the direct $\epsilon_{\text {micro }}-\epsilon_{\text {fine }}$ relationship
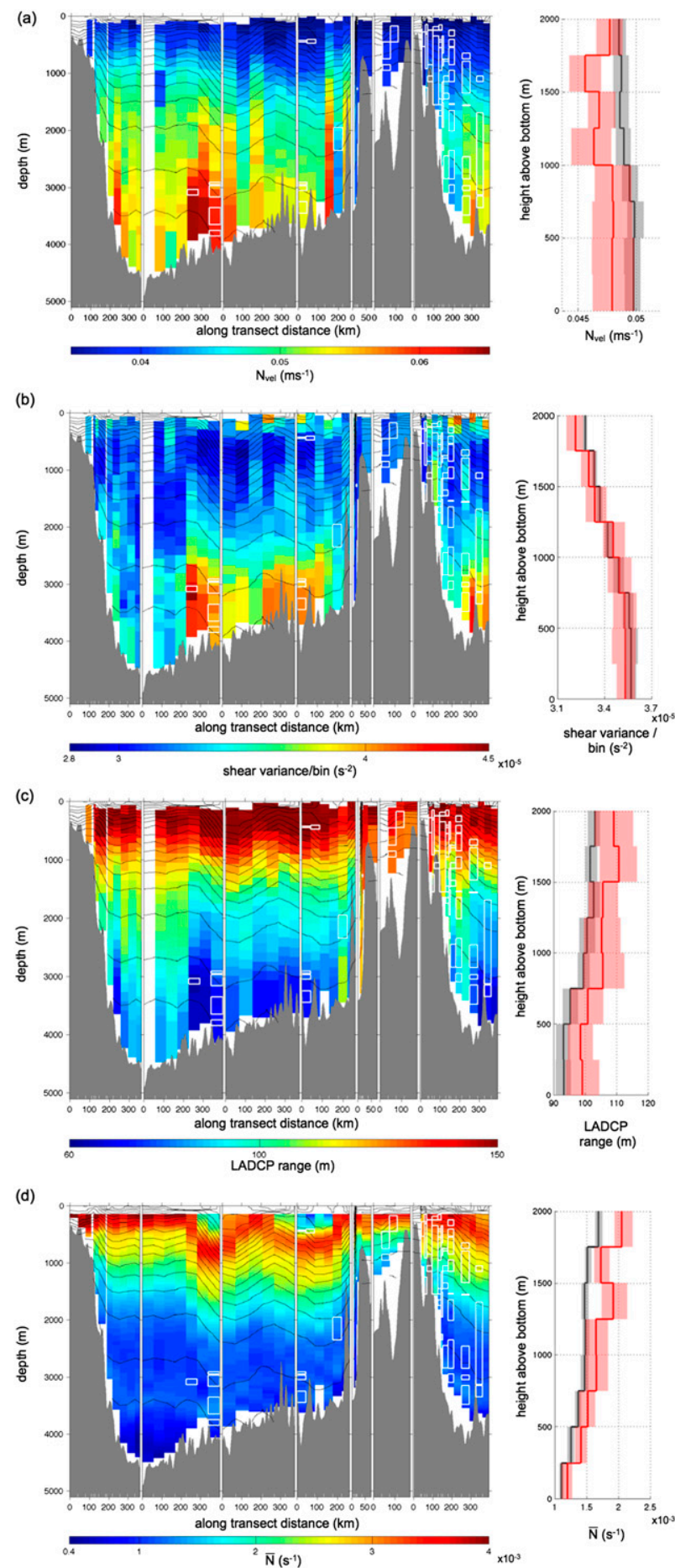

FIG. A1. Spatial distributions and station-averaged vertical profiles as a function of height of four select LADCP noise diagnostics: (a) nominal noise level $N_{\text {vel }}$; (b) shear variance per bin; (c) LADCP range; and (d) mean stratification $\bar{N}$ averaged in 640m-depth bins common to the $\epsilon_{\text {fine }}$ calculation. In each, the alongtransect distance-depth sections are displayed as in Fig. 2. White outlines mark the locations where the $\epsilon$ ratio exceeds 5 and as such locations of anomalously large finescale overprediction. Vertical profiles contrast the overall (all station) mean (black) with the average of the special eight stations (red). The shaded areas show the $90 \%$ confidence interval calculated by bootstrapping. 
(a)

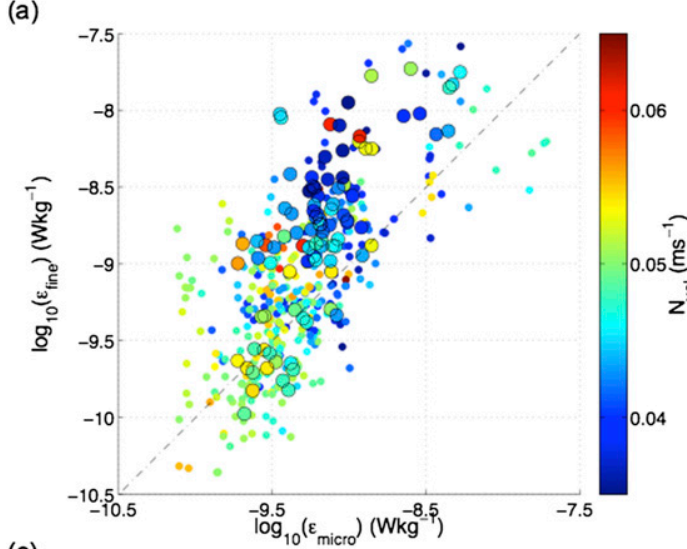

(c)

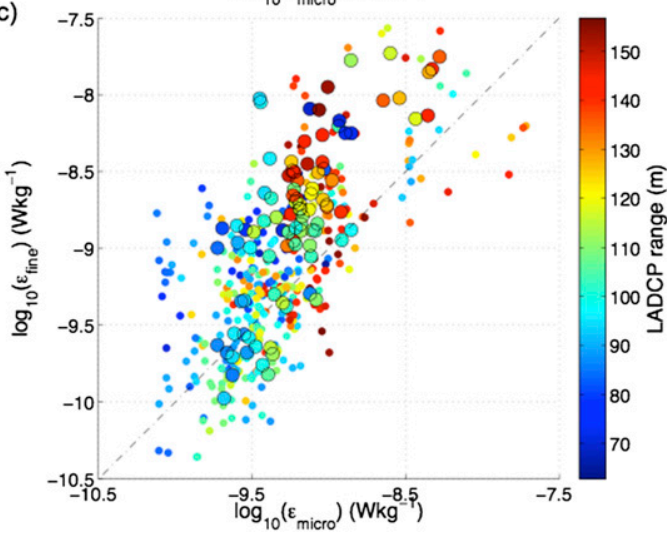

(b) -7.5

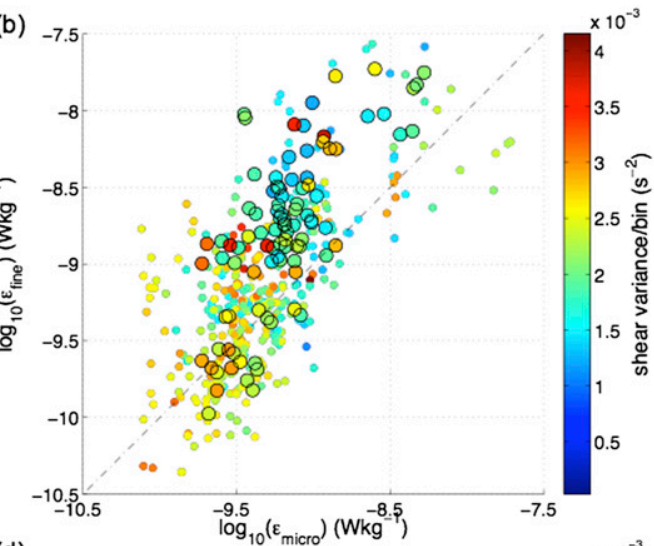

(d)

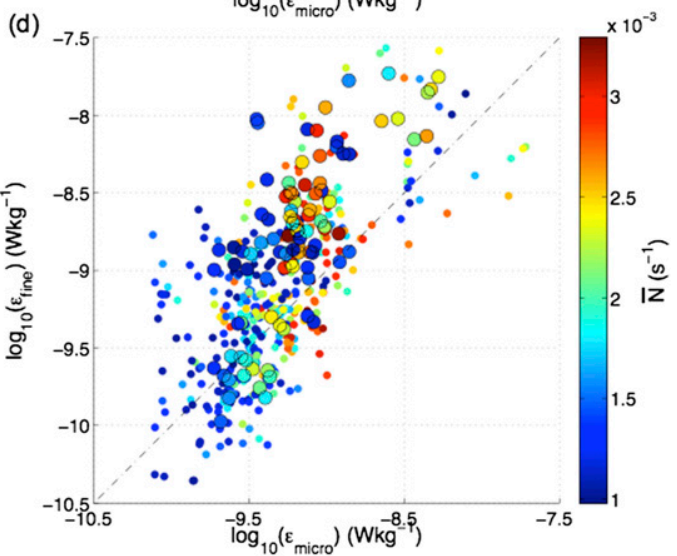

FIG. A2. The relationship between $\epsilon_{\text {micro }}$ and $\epsilon_{\text {fine }}$ colored by select LADCP noise diagnostics: (a) nominal noise level $N_{\text {vel }}$; (b) shear variance per LADCP bin; (c) LADCP range; and (d) mean stratification $\bar{N}$ averaged in depth bins defined by the $\epsilon_{\text {fine }}$ calculation. Display is as in Fig. 9a.

(Fig. A2) shows a tendency for measures of low dissipation rates to be associated with higher nominal noise levels, higher values of shear variance/bin, lower LADCP ranges, and lower stratifications. Given that the instances of finescale overprediction tend to be associated with higher values of the dissipation rate, they therefore do not tend to occur in association with the highest relative noise levels, consistent with the average vertical profiles in Fig. A1 indicating that the large near-bottom overpredictions are associated with lower noise levels than the overall average. An examination of the direct dependence of the $\epsilon$ ratio on these noise diagnostics (Fig. A3) reveals no significant correlation between this relationship and any of the noise diagnostics considered. Further, the analysis displayed in both Figs. A2 and A3 shows no systematic tendency for the $\epsilon_{\text {fine }}$ estimates for the special eight stations to be associated with relatively high or anomalous noise levels.

(iii) Near-bottom shear spectra and their relation to the implied energy spectrum of the LADCP noise grouped as a function of nominal noise level (Fig. A4) indicate that as the nominal noise level is increased, the vertical scale for which we expect LADCP noise to significantly contaminate the shear variance level (that for which the average spectra adopts the shape of the noise spectrum) also is increased, an expected result. However, even for locations where the noise level is extreme (nominal noise level greater than the mean level plus one standard deviation), this vertical scale is significantly smaller than the average scale corresponding to $m_{c}$ for the group (thick vertical lines in Fig. A4) and also smaller than the scales considered by our typical implementations of the finescale parameterization (dashed vertical lines in Fig. A4). Further, near-bottom spectral shape at these "worst case" locations does not appear anomalous or present specific cause for concern: the shape of the shear and strain spectra do not appear particularly unusual relative to the $m_{c} / m$ roll off, nor the nominal high-wavenumber asymptote of the GM spectrum. A differentiation of $\mathrm{CW}$ - versus $\mathrm{CCW}$-polarized 

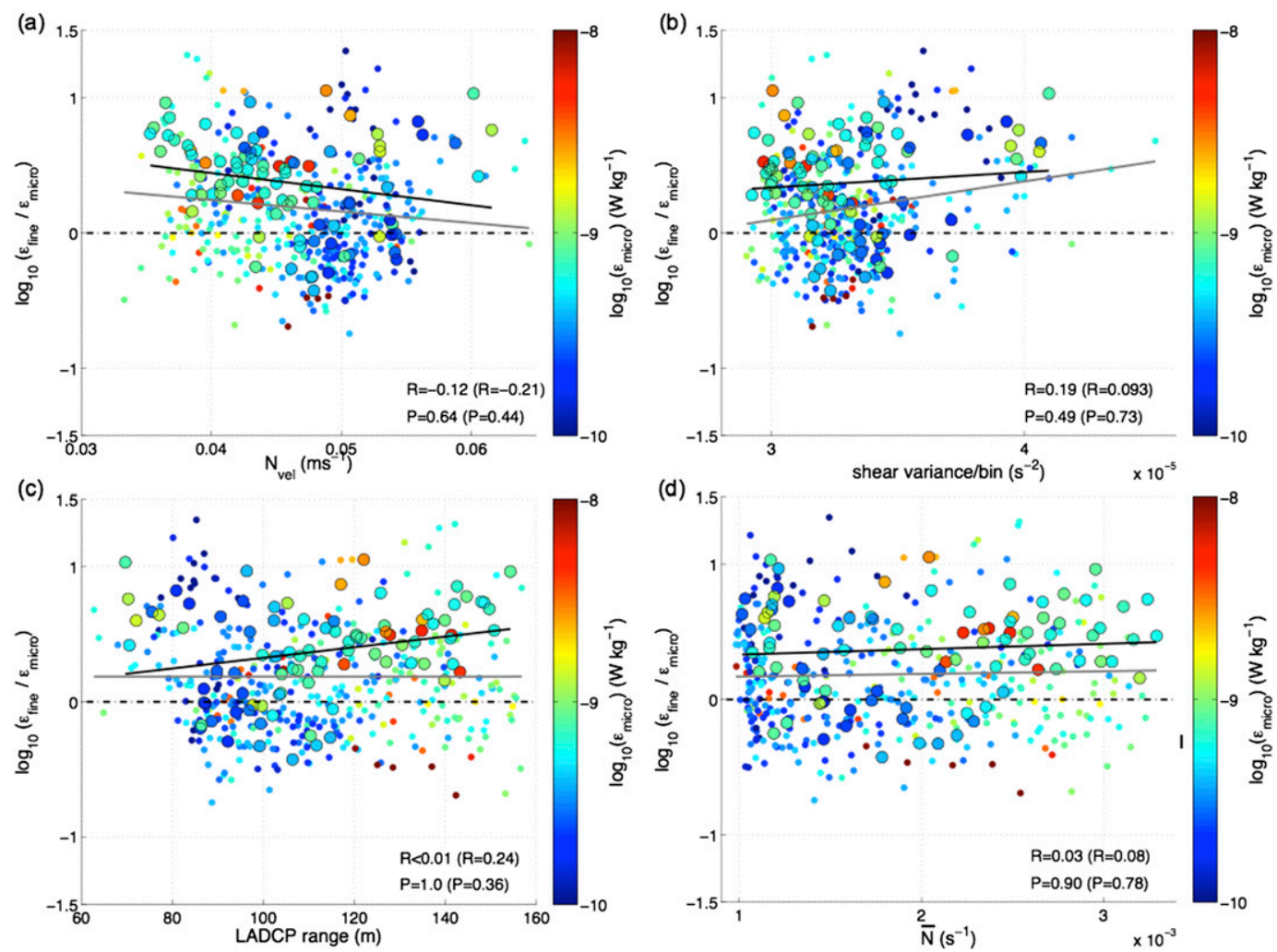

FIG. A3. Relation between select LADCP noise diagnostics and finescale overprediction: (a) nominal noise level $N_{\text {vel }}$; (b) shear variance per LADCP bin; (c) LADCP range; and (d) mean stratification $\bar{N}$ averaged in depth bins defined by the $\epsilon_{\text {fine }}$ calculation. Display is as in Fig. 13.

shear variance at the length scales considered by the finescale parameterization (and significantly smaller) for all the average near-bottom rotary spectra, including those for stations characterized by extreme noise levels (Fig. A4b), further suggests that the near-bottom spectra are not significantly contaminated by noise (which we expect to have no distinct polarization) at the wavelengths under consideration.

(iv) Finally, a comparison of the average shear and rotary spectra for locations where the finescale parameterization over- versus underpredicts (Figs. A4c,d) shows that while there is a systematic difference in the shear variance level for locations of finescale over- versus underprediction (with overpredicting stations being associated with higher shear variance levels at all vertical wavelengths down to those that are dominated by noise), there is not a significant difference in shear spectra shape, with the average spectral shape for both groups being very similar. We note also that the variance level of the average implied energy spectrum of the LADCP noise (dashed-dotted lines in Fig. A4c) for the overpredicting locations is in fact lower than the average noise level for underpredicting locations, again consistent with the observation that the overpredicting stations tend to be associated with a lower nominal noise level at depth. As such, the vertical wavelength at which we expect noise to contaminate the shear variance level at stations that overpredict is smaller than that which characterizes the locations that tend to underpredict. Average rotary spectra for both over- and underpredicting depth bins show differentiation in the $\mathrm{CCW}$ - versus $\mathrm{CW}$-polarized shear variance at vertical scales significantly smaller than those considered by the finescale parameterization, again suggesting that the shear variance measurements at the scales considered are physical and not dominated by noise.

In summary, a diverse collection of analyses provide no suggestion that the near-bottom finescale parameterization overprediction that we observe in key places is linked to spurious signals in the LADCP velocity finestructure observations arising from measurement noise and/or processing procedures. Instead, they suggest that in fact the locations of most extreme near-bottom overprediction 
(a)

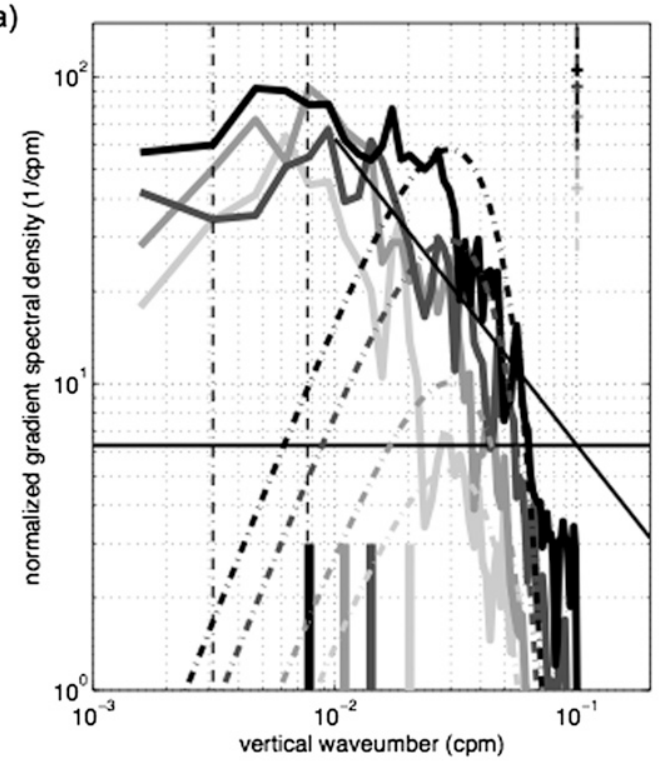

(c)

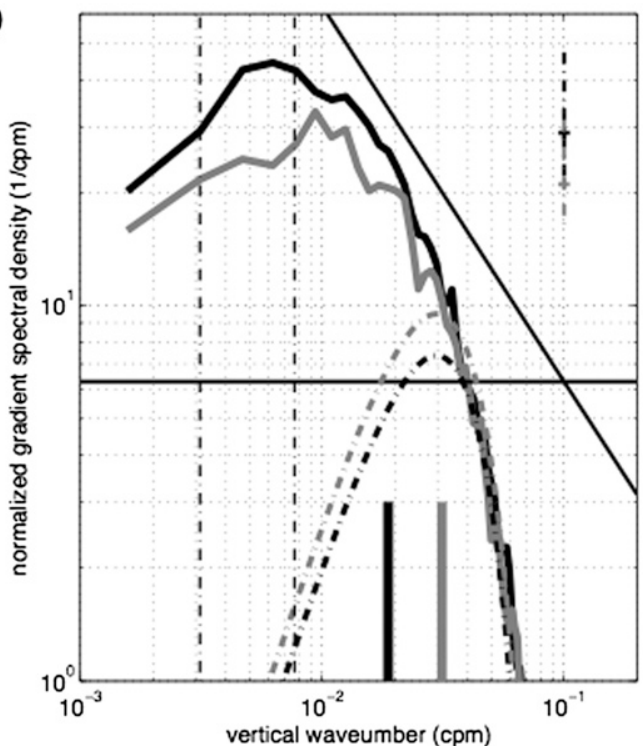

(b)

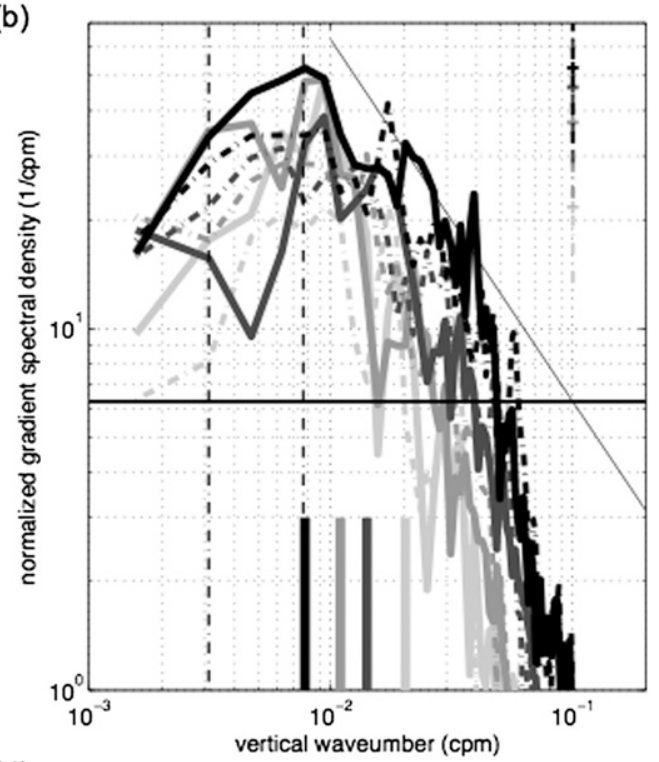

(d)

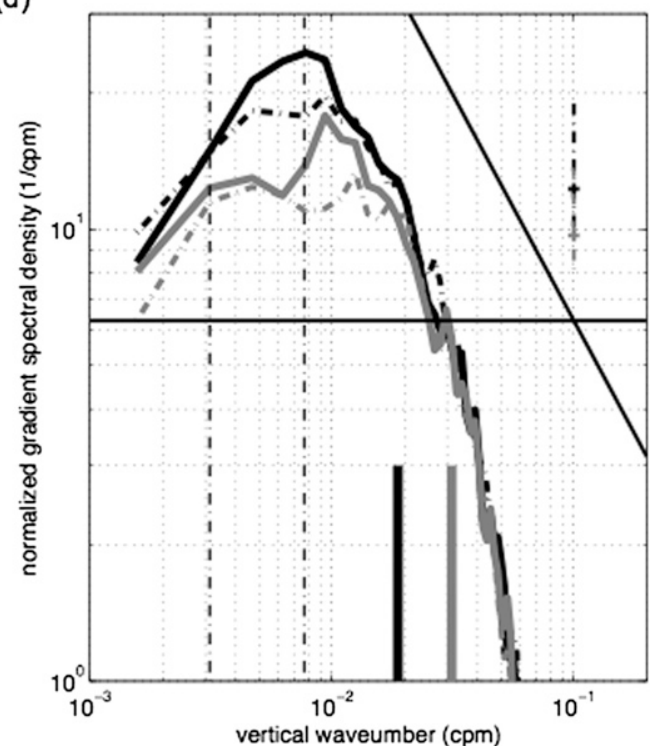

FIG. A4. (a) Mean buoyancy frequency-normalized shear spectra for the bottommost transform bin (within $640 \mathrm{~m}$ of the bottom) for groupings based on the nominal noise level: increasingly darker shades of gray denote stations having nominal noise levels characterized as very low, below average, above average, and very high, where very low and very high refer to values less than or greater than the median level plus or minus one std dev, respectively, and below and above average refer to values between the median level plus or minus one std dev. Dashed-dotted thick lines show the LADCP noise model of Polzin et al. (2002) based on the average number of pings in each depth bin for each grouping and 10 times the nominal noise level of $0.032 \mathrm{~m} \mathrm{~s}^{-1}$ in each beam pair. The thick vertical lines indicate the average value of $m_{c}$ computed from the shear variance level of the average shear spectrum for each of the station groupings. The nominal high-wavenumber asymptote of the GM spectrum (horizontal black line) and the saturation spectra (thin black line) are shown for reference. The confidence intervals indicate the $95 \%$ confidence interval that is proportional to the number of stations in each grouping. (b) As in (a), but showing the average $\mathrm{CW}$-polarized component of the buoyancy frequency-normalized shear (thick solid lines) and CCW-polarized component (thick dashed-dotted lines). (c),(d) As in (a),(b), but for stations grouped based on their tendency for finescale overprediction (black) vs underprediction (gray). Here all depths are considered. 
(the special eight stations) are characterized by lower average noise levels than the dataset as a whole. The observed shear spectra, even in the worst case scenarios of extreme noise levels and/or large finescale parameterization overprediction, are well in excess of 10 times the theoretical noise spectra, and a distinctly polarized shear appears fully resolved at the vertical wavelengths used in all implementations of the parameterization. They provide a strong case that LADCP instrumental noise and LADCP data processing artifacts are not the key underpinning cause of the overprediction signal observed.

\section{APPENDIX B}

\section{Lee-Wave Evolution in a Backing Vertical Shear}

Here we consider the expected evolution of the vertical wavenumber of a lee wave in a backing, vertically sheared flow. The aim is to evaluate whether the scales of the vertical wavenumber and inferred wave frequency we observe at the off-bottom height are consistent with the expected evolution of a lee wave generated at the bottom. Specifically we ask the following:

(i) What change in vertical wavenumber $m$ do we expect to see at a fixed height above the bottom in the observed background flow shear assuming a lee-wave characterization [i.e., a particular horizontal wavenumber $k$ and a wave frequency $\omega(z)=-k U(z)$, where $U$ is the magnitude of the background flow and $z$ is the vertical height coordinate]?

(ii) Is this expected change consistent with the relation between the observed scale of $m$ at height and our expectation for the lee-wave vertical wavenumber given the observed bottom flow speed and stratification?

The internal wave dispersion relation dictates (here assuming $N^{2} \gg \omega^{2}$ )

$$
\frac{k^{2}}{m^{2}}=\frac{\omega^{2}-f^{2}}{N^{2}} .
$$

Here $k$ is the horizontal wavenumber characterizing the lee wave in question [we assume a rotation of the horizontal coordinate system so that it is aligned in the direction of the local velocity vector and a one-dimensional effective topographic spectrum of the form $P(k)$; see Nikurashin and Ferrari (2011) for further details], $m$ is the lee-wave vertical wavenumber, $\omega$ is the lee-wave intrinsic frequency, $f$ is the local inertial frequency, and $N$ is the buoyancy frequency characterizing the background stratification.

The dispersion relation [Eq. (B1)] holds at every height so the central vertical wavenumber of a lee-wave packet at an off-bottom height can be estimated via

$$
\frac{m^{2}}{m_{0}^{2}}=\frac{\omega_{0}^{2}-f^{2}}{\omega^{2}-f^{2}} .
$$

Here a subscript of 0 denotes the value at $z=0$. Otherwise, the intrinsic frequency, vertical wavenumber, and background velocity are considered to be depth dependent. For simplicity we take the near-bottom stratification $N$ to be constant. Substituting the observed values of $\omega(1000 \mathrm{~m}) \sim 1.1 f$ (inferred from the observed $R_{\omega}$ profile) and $U_{0} /[U(1000 \mathrm{~m})] \sim 2$ (inferred from Fig. 14a) into Eq. (B2) gives an expected ratio of $m(1000 \mathrm{~m})$ to $m_{0}$ of $\sim 18$; that is, the vertical wavelength of this wave is expected to be 18 times smaller at $z=$ $1000 \mathrm{~m}$ than at the bottom boundary.

We compare the ratio of the scale of the observed wavenumber $m$ at $z=1000 \mathrm{~m}$ [i.e., $m(1000 \mathrm{~m}) \sim$ $2 \pi /(100 \mathrm{~m})]$ to the expected lee-wave vertical wavenumber at the bottom, given the observed scales of the near-bottom flow magnitude and the background stratification. Taking $U_{0} \sim O\left(0.2 \mathrm{~m} \mathrm{~s}^{-1}\right)$ and $N \sim$ $O\left(0.001 \mathrm{~s}^{-1}\right)$ returns $m_{0}=N / U_{0} \sim 0.005 \mathrm{~m}^{-1}$. The ratio of $m(1000 \mathrm{~m}) / m_{0}$ for the observed off-bottom vertical wavenumber and the anticipated vertical wavenumber at the bottom assuming a lee-wave characterization is therefore $\sim 12$.

The consistent orders for the expected ratio of vertical wavenumbers at $z=1000 \mathrm{~m}$ and $z=0 \mathrm{~m}$ thus shows that the observed scales are consistent with the expected evolution of a lee wave toward a critical layer situation. This lends support to the hypothesis that wave-mean flow interactions play a key role in the evolution of the waves in question at the sites prone to large finescale overprediction.

This characterization of the typical wave properties at the special sites both at the bottom and at the off-bottom height of $z=1000 \mathrm{~m}$, assuming a lee-wave characterization, also permits us to estimate both the horizontal and vertical components of the typical wave's group velocity at these two levels under this assumption. Making the typical near-inertial and hydrostatic approximations such that these components are given by $c_{g H}=$ $\left[N\left(\omega^{2}-f^{2}\right)^{1 / 2}\right] / \omega m$ and $c_{g z}=\left[\left(\omega^{2}-f^{2}\right)^{3 / 2}\right] / \omega N k \quad($ Gill 1982), and then substituting $\omega_{0}=-k U_{0}$ and $k=$ $-[2 \omega(1000 \mathrm{~m})] / U_{0}$ based on the arguments above, yields scale estimates for $c_{g H}$ at $z=0$ of $O\left(10 \mathrm{~cm} \mathrm{~s}^{-1}\right)$ and at $z=1000 \mathrm{~m}$ of $O\left(1 \mathrm{~cm} \mathrm{~s}^{-1}\right)$ and scale estimates for $c_{g z}$ at $z=0$ of $O\left(1 \mathrm{~cm} \mathrm{~s}^{-1}\right)$ and at $z=1000 \mathrm{~m}$ of $O\left(0.1 \mathrm{~cm} \mathrm{~s}^{-1}\right)$. Assuming the bottommost $1000 \mathrm{~m}$ is characterized by $c_{g H}$ and $c_{g z}$ values of 5 and $0.5 \mathrm{~cm} \mathrm{~s}^{-1}$, respectively, and that the background flow in this height range is characterized by a value of $15 \mathrm{~cm} \mathrm{~s}^{-1}$, this implies that a wave generated at the bottom will propagate in the horizontal $\sim 10 \mathrm{~km}$ and be advected by 
the background flow a farther $\sim 30 \mathrm{~km}$ in the time it propagates to $1000-\mathrm{m}$ height. These scale estimates thus provide quantitative support for the claim that horizontal wave propagation and generating flow advection play significant roles in the evolution of a lee wave between its generation at the bottom and its arrival at the height where the background shear changes sign and its evolution toward critical layer properties is halted. Further, they suggest that considering onedimensional vertical profiles of wave and turbulent properties as records of the wave's evolution as it propagates upward from the bottom relies on the statistical homogeneity of the lee-wave generation process.

\section{REFERENCES}

Bell, T. H., 1975: Lee waves in stratified flows with simple harmonic time dependence. J. Fluid Mech., 67, 705-722, doi:10.1017/ S0022112075000560.

Brearley, J. A., A. C. Naveira Garabato, K. Sheen, D. Smeed, and S. Waterman, 2013: Eddy-induced modulation of turbulent dissipation over rough topography in the Southern Ocean. J. Phys. Oceanogr., 43, 2288-2308, doi:10.1175/ JPO-D-12-0222.1.

Eriksen, C. C., 1985: Implications of ocean bottom reflection for internal wave spectra and mixing. J. Phys. Oceanogr., 15, 1145-1156, doi:10.1175/1520-0485(1985)015<1145: IOOBRF $>2.0 . \mathrm{CO} ; 2$.

Firing, E., and R. Gordon, 1990: Deep ocean acoustic Doppler current profiling. Proc. IEEE Fourth Working Conf. on Current Measurement, Clinton, MD, IEEE, 192-201, doi:10.1109/ CURM.1990.110905.

Fischer, J., and M. Visbeck, 1993: Deep velocity profiling with selfcontained ADCPs. J. Atmos. Oceanic Technol., 10, 764-773, doi:10.1175/1520-0426(1993)010<0764:DVPWSC >2.0.CO;2.

Gargett, A. E., 1990: Do we really know how to scale the turbulent kinetic energy dissipation rate $\epsilon$ due to breaking of oceanic internal waves? J. Geophys. Res., 95 (C9), 15971-15974, doi:10.1029/JC095iC09p15971.

Gill, A. E., 1982: Atmosphere-Ocean Dynamics. Academic Press, $662 \mathrm{pp}$.

Gregg, M. C., 1989: Scaling turbulent dissipation in the thermocline. J. Geophys. Res., 94 (C7), 9686-9698, doi:10.1029/ JC094iC07p09686.

— Monica Basin. J. Geophys. Res., 96 (C9), 16709-16719, doi:10.1029/91JC01385.

_ , T. B. Sanford, and D. P. Winkel, 2003: Reduced mixing from the breaking of internal waves in equatorial ocean waters. Nature, 422, 513-515, doi:10.1038/nature01507.

Henyey, F. S., 1991: Scaling of internal wave predictions for $\epsilon$. Dynamics of Internal Gravity Waves in the Ocean: Proc. 'Aha Huliko'a Hawaiian Winter Workshop, Honolulu, HI, University of Hawai'i at Mānoa, 233-236.

__ J. Wright, and S. Flatte, 1986: Energy and action flow through the internal wave field: An eikonal approach. J. Geophys. Res., 91 (C7), 8487-8496, doi:10.1029/JC091iC07p08487.

King, B. A., E. Firing, and T. M. Joyce, 2001: Shipboard observations during WOCE. Ocean Circulation and Climate: $\mathrm{Ob}$ serving and Modeling the Global Ocean, G. Siedler, J. Church, and J. Gould, Eds., Academic Press, 99-122.
Kunze, E., A. J. Williams III, and M. G. Briscoe, 1990: Observations of shear and vertical stability from a neutrally buoyant float. J. Geophys. Res., 95 (C10), 18127-18142, doi:10.1029/ JC095iC10p18127.

, L. K. Rosenfeld, G. S. Carter, and M. C. Gregg, 2002: Internal waves in Monterey submarine canyon. J. Phys. Oceanogr., 32, 1890-1913, doi:10.1175/1520-0485(2002)032<1890: IWIMSC $>2.0 . \mathrm{CO} ; 2$

—, E. Firing, J. M. Hummon, T. K. Chereskin, and A. M. Thurnherr, 2006: Global abyssal mixing inferred from lowered ADCP shear and CTD strain profiles. J. Phys. Oceanogr., 36, 1553-1576, doi:10.1175/JPO2926.1.

Mauritzen, C., K. L. Polzin, M. S. McCartney, R. C. Millard, and D. E. West-Mack, 2002: Evidence in hydrography and density fine structure for enhanced vertical mixing over the MidAtlantic Ridge in the western Atlantic. J. Geophys. Res., 107, 3147, doi:10.1029/2001JC001114.

Muller, P., and N. Xu, 1992: Scattering of oceanic internal gravity waves off random bottom topography. J. Phys. Oceanogr., 22, 474-488, doi:10.1175/1520-0485(1992)022<0474: SOOIGW $>2.0 . \mathrm{CO} ; 2$.

Munk, W., 1981: Internal waves and small-scale process. Evolution of Physical Oceanography, B. A. Warren and C. A. Wunsch, Eds., MIT Press, 264-291.

Naveira Garabato, A. C., 2009: RRS James Cook Cruise 29, 01 Nov-22 Dec 2008. SOFine Cruise Rep.: Southern Ocean Finestructure, National Oceanography Centre Southampton Cruise Rep. 35, 216 pp.

—, K. Polzin, B. King, K. Heywood, and M. Visbeck, 2004: Widespread intense turbulent mixing in the Southern Ocean. Science, 303, 210-213, doi:10.1126/science.1090929.

_ A. J. G. Nurser, R. B. Scott, and J. A. Goff, 2013: The impact of small-scale topography on the dynamical balance of the ocean. J. Phys. Oceanogr., 43, 647-668, doi:10.1175/JPO-D-12-056.1.

Nikurashin, M., and R. Ferrari, 2010a: Radiation and dissipation of internal waves generated by geostrophic motions impinging on small-scale topography: Application to the Southern Ocean. J. Phys. Oceanogr., 40, 2025-2042, doi:10.1175/ 2010JPO4315.1.

$\longrightarrow$, and - 2010b: Radiation and dissipation of internal waves generated by geostrophic flows impinging on small-scale topography: Theory. J. Phys. Oceanogr., 40, 1055-1074, doi:10.1175/2009JPO4199.1.

$\longrightarrow$, and - 2011: Global energy conversion rate from geostrophic flows into internal lee waves in the deep ocean. Geophys. Res. Lett., 38, L08610, doi:10.1029/2011GL046576.

Oakey, N. S., 1982: Determination of the rate of dissipation of turbulent energy from simultaneous temperature and velocity shear microstructure measurements. J. Phys. Oceanogr., 12, 256-271, doi:10.1175/1520-0485(1982)012<0256: DOTROD $>2.0 . \mathrm{CO} ; 2$.

Polzin, K. L., 2004: Idealized solutions for the energy balance of the finescale internal wave field. J. Phys. Oceanogr., 34, 231-246, doi:10.1175/1520-0485(2004)034<0231:ISFTEB >2.0.CO;2. 2009: An abyssal recipe. Ocean Modell., 30, 298-309, doi:10.1016/j.ocemod.2009.07.006.

2010: Mesoscale eddy-internal wave coupling. Part II: Energetics and results from PolyMode. J. Phys. Oceanogr., 40, 789-801, doi:10.1175/2009JPO4039.1.

, and E. T. Montgomery, 1996: Deep microstructure profiling with the High Resolution Profiler. Proc. Microstructure Sensors Workshop, Mt. Hood, OR, Office of Naval Research, 109-115. 
, and Y. L. Lvov, 2011: Toward regional characterizations of the oceanic internal wavefield. Rev. Geophys., 49, RG4003, doi:10.1029/2010RG000329.

_ J. M. Toole, and R. W. Schmitt, 1995: Finescale parameterizations of turbulent dissipation. J. Phys. Oceanogr., 25, 306-328, doi:10.1175/1520-0485(1995)025<0306:FPOTD>2.0.CO;2.

, E. Kunze, J. Hummon, and E. Firing, 2002: The finescale response of lowered ADCP velocity profiles. J. Atmos. Oceanic Technol., 19, 205-223, doi:10.1175/1520-0426(2002)019<0205: TFROLA $>2.0 . \mathrm{CO} ; 2$.

A. C. N. Garabato, T. N. Huussen, B. M. Sloyan, and S. Waterman, 2014: Finescale parameterizations of turbulent dissipation. J. Geophys. Res. Oceans, 119, 1383-1419, doi:10.1002/2013JC008979.

Scott, R. B., J. A. Goff, A. C. N. Garabato, and A. J. G. Nurser, 2011: Global rate and spectral characteristics of internal gravity wave generation by geostrophic flow over topography. J. Geophys. Res., 116, C09029, doi:10.1029/2011JC007005.

Sheen, K. L., and Coauthors, 2013: Rates and mechanisms of turbulent dissipation and mixing in the Southern Ocean: Results from the Diapycnal and Isopycnal Mixing Experiment in the Southern Ocean experiment. J. Geophys. Res. Oceans, 118, 2774-2792, doi:10.1002/jgrc.20217.
Sloyan, B. M., 2005: Spatial variability of mixing in the Southern Ocean. Geophys. Res. Lett., 32, L18603, doi:10.1029/2005GL023568.

Smith, W. H. F., and D. T. Sandwell, 1997: Global seafloor topography from satellite altimetry and ship depth soundings. Science, 277, 1956-1962, doi:10.1126/science.277.5334.1956.

Sokolov, S., and S. R. Rintoul, 2009: Circumpolar structure and distribution of the Antarctic Circumpolar Current fronts: 1. Mean circumpolar paths. J. Geophys. Res., 114, C11018, doi:10.1029/2008JC005108.

Sparrow, M. D., K. J. Heywood, J. Brown, and D. P. Stevens, 1996 Current structure of the southern Indian Ocean. J. Geophys. Res., 101 (C3), 6377-6391, doi:10.1029/95JC03750.

Visbeck, M., 2002: Deep velocity profiling using lowered acoustic Doppler current profilers: Bottom track and inverse solutions. J. Atmos. Oceanic Technol., 19, 794-807, doi:10.1175/ 1520-0426(2002)019<0794:DVPULA >2.0.CO;2.

Waterman, S., A. C. Naveira Garabato, and K. L. Polzin, 2013: Internal waves and turbulence in the Antarctic Circumpolar Current. J. Phys. Oceanogr., 43, 259-282, doi:10.1175/JPO-D-11-0194.1.

Wu, L., Z. Jing, S. Riser, and M. Visbeck, 2011: Seasonal and spatial variations of Southern Ocean diapycnal mixing from Argo profiling floats. Nat. Geosci., 4, 363-366, doi:10.1038/ ngeo1156. 